\title{
A FEW SYMMETRY RESULTS FOR NONLINEAR ELLIPTIC PDE ON NONCOMPACT MANIFOLDS
}

\author{
Luís ALMEIDA ${ }^{\mathrm{a}}$, Lucio DAMASCELLI ${ }^{\mathrm{b}}$, Yuxin GE $^{\mathrm{c}}$ \\ ${ }^{a}$ Laboratoire J.A. Dieudonné - CNRS (UMR 6621), Université de Nice - Sophia-Antipolis, Parc Valrose, \\ F-06108 Nice Cedex 02, France \\ b Dipartimento di Matematica, Università di Roma "Tor Vergata", Via della Ricerca Scientifica, \\ 00133 Roma, Italy \\ ${ }^{\mathrm{c}}$ Département de Mathématiques, Faculté de Sciences et Technologie, Université Paris XII-Val de Marne, \\ 61, avenue du Général de Gaulle, 94010 Créteil Cedex, France
}

Received 11 January 2001, revised 4 May 2001

\begin{abstract}
We prove some symmetry theorems for positive solutions of elliptic equations in some noncompact manifolds, which generalize and extend symmetry results known in the case of the euclidean space $\mathbb{R}^{n}$. The (variational) technique that we use relies on Sobolev inequalities available for manifolds together with the well known method of moving planes. In the particular case of the standard $n$-dimensional hyperbolic space $\mathbb{H}^{n}$ we get the radial symmetry of positive solutions of the equation $-\Delta_{\mathbb{H}^{n}} u=f(u)$ in $\mathbb{H}^{n}$, which tend to zero at infinity (or belong to the Sobolev space $H^{1}\left(\mathbb{H}^{n}\right)$ in some cases), under different hypotheses on the relationship between the behavior of the nonlinearity $f$ in a neighborhood of zero and the summability properties of the solution. One of the main features of this work is to single out and study the connection between the geometric properties of the manifold considered and the growth conditions on the nonlinearity in order to have our symmetry results.
\end{abstract}

(C) 2002 L'Association Publications de l'Institut Henri Poincaré. Published by Elsevier B.V. All rights reserved

AMS classification: 35J50; 35J60; 35Q35; 58E05

RÉSUMÉ. - Nous démontrons quelques résultats de symétrie pour des solutions positives de certaines équations différentielles partielles sur des variétés - ce sont des généralisations de résultats qui étaient déjà connus dans le cas des espaces euclidien, $\mathbb{R}^{n}$. La technique variationnelle est basée sur des inégalités de Sobolev dans le cadre des variétés, combinées avec une adaptation de la méthode traditionnelle de "moving planes" à notre situation. En particulier, dans le cas de l'espace hyperbolique à $n$ dimensions, $\mathbb{H}^{n}$, nous démontrons la symétrie radiale des solutions positives de $-\Delta u=f(u)$ dans $\mathbb{H}^{n}$, qui tendent vers zéro à l'infini (ou, dans certains cas, appartiennent à l'espace de Sobolev $\left.H^{1}\left(\mathbb{H}^{n}\right)\right)$.

Un des intérêts majeurs de ce travail réside dans les relations mises en évidence entre certaines caractéristiques de nature géométrique de la variété domaine et les propriétés de nature analytique

E-mail addresses: luis@math.unice.fr (L. Almeida), damascel@mat.uniroma2.it (L. Damascelli), ge@univ-paris12.fr (Y. Ge). 
(taux de croissance) des non linéarités admissibles.

(C) 2002 L'Association Publications de l'Institut Henri Poincaré. Published by Elsevier B.V. All rights reserved

\section{Introduction}

In this paper we prove some symmetry results for positive solutions of elliptic equations in noncompact manifolds. For the euclidean space $\mathbb{R}^{n}$ the problem was first studied by Gidas, $\mathrm{Ni}$ and Nirenberg [11] using the method of moving planes of Alexandrov [1] and Serrin [16] (see also the works of Gidas, Ni and Nirenberg [10] and Berestycki and Nirenberg [7] concerning the use of this method for symmetry problems in bounded domains). Among other results, they prove that $C^{2}$ solutions of the problem

$$
\begin{cases}-\Delta u=f(u), u>0 & \text { in } \mathbb{R}^{n}, \\ u \rightarrow 0 & \text { when }|x| \rightarrow \infty\end{cases}
$$

are radial provided $f \in C^{1+\alpha}[0, \infty), f(0)=0, f^{\prime}(0)<0$. They also obtain certain symmetry results in the case $f^{\prime}(0)=0$ under appropriate assumptions on the growth of $f$ near 0 and the decay of $u$ at $\infty$. These results were then extended by $\mathrm{Li}$ and $\mathrm{Ni}$ [15] who proved the symmetry of the solutions of (1) under the hypothesis

$$
\exists s_{0}>0: f^{\prime}(s) \leqslant 0 \quad \forall s \in\left(0, s_{0}\right) .
$$

Moreover, the work of Li [14] extended the symmetry results to fully nonlinear strictly elliptic equations - in particular he obtained the symmetry of solutions of (1) when

$$
f^{\prime}(s)=\mathrm{O}\left(s^{\alpha}\right), s \rightarrow 0 \text { for } \alpha>0 ; \quad u=\mathrm{O}\left(\frac{1}{|x|^{m}}\right),|x| \rightarrow \infty, \text { and } m \alpha>2 .
$$

Note that in this case $u \in L^{\alpha \frac{n}{2}}\left(\mathbb{R}^{n}\right)$.

In fact, as we show in Section 2, it is possible to obtain this symmetry result for $\mathbb{R}^{n}$ under the sole assumption that $u$ belongs to the space $L^{\alpha \frac{n}{2}}\left(\mathbb{R}^{n}\right)$ using Sobolev inequalities - this will be the technique that we will then extend in order to prove symmetry results in the case of more general noncompact manifolds.

Serrin and Zou [17] studied the symmetry of positive solutions of general quasilinear elliptic equations - in particular, for (1) their results yield the symmetry of solutions if $f$ is locally Lipschitz continuous in $(0, \infty)$ and nonincreasing near zero.

In [8] a symmetry result has been proved for positive solutions $u \in W^{1, p}\left(\mathbb{R}^{n}\right) \cap C^{1}\left(\mathbb{R}^{n}\right)$ of the problem analogous to (1), when the laplacian is replaced by the $p$-Laplace operator, $1<p<2$ and $f$ is a locally Lipschitz continuous function in $(0, \infty)$, nonincreasing near zero. Recently [9] this result was extended to solutions which belong only to the space $C^{1}\left(\mathbb{R}^{n}\right)$ assuming $f$ is nonincreasing near zero, and the analogous of C. Li's result for the $p$-laplacian has been proved, when the behavior of the solution at infinity is known, exploiting Poincaré and Hardy inequalities.

Our technique is inspired by the same philosophy, since it relies on different Sobolev type inequalities available for manifolds. 
After the paper was completed we learned that the technique exploiting Sobolev inequalities together with the moving plane method was used by Terracini $[18,19]$ in two elegant papers where singular problems in $\mathbb{R}^{n}$ and elliptic problems in half spaces with nonlinear boundary conditions were studied.

We also remark that a thorough study of symmetry and monotonicity properties of solutions of elliptic equations in unbounded domains distinct from $\mathbb{R}^{n}$, like half spaces of cylinders, has been carried out by Berestycki, Caffarelli and Nirenberg in [3-6].

For the reader's convenience, we will first prove, using our technique, the (slight generalization of) some known results for the $\mathbb{R}^{n}$ setting, namely

THEOREM 1. - Let $u \in C^{1}\left(\mathbb{R}^{n}\right), n \geqslant 3$, be a (weak) solution of the equation

$$
\begin{cases}-\Delta u=f(u) & \text { in } \mathbb{R}^{n}, \\ u>0 & \text { in } \mathbb{R}^{n},\end{cases}
$$

where $f$ is locally Lipschitz continuous in $(0, \infty)$. Then $u$ is radially symmetric and strictly radially decreasing around some point $x_{0} \in \mathbb{R}^{n}$ provided one of the following holds:

(a) $u(x) \rightarrow 0$ as $|x| \rightarrow \infty$ and $\exists s_{0}>0$ such that $f$ is nonincreasing in $\left(0, s_{0}\right)$;

(b) $u(x) \rightarrow 0$ as $|x| \rightarrow \infty, \exists s_{0}, \alpha>0$ such that if $0<a<b<s_{0}$ then $\frac{f(b)-f(a)}{b-a} \leqslant$ $C(a+b)^{\alpha}$ and $u \in L^{\alpha \frac{n}{2}}\left(\mathbb{R}^{n}\right)$;

(c) $u \in \mathcal{D}^{1,2}\left(\mathbb{R}^{n}\right):=\left\{u \in L^{2^{*}}\left(\mathbb{R}^{n}\right):|D u| \in L^{2}\left(\mathbb{R}^{n}\right)\right\}$, where $2^{*}=\frac{2 n}{n-2}, \quad \exists \alpha>0$ such that if $0<a<b$ then $\left|\frac{f(b)-f(a)}{b-a}\right| \leqslant C(a+b)^{\alpha}$ and $u \in L^{\alpha \frac{n}{2}}\left(\mathbb{R}^{n}\right)$.

Remarks. - 1) Note that in the critical case, i.e. when $\alpha=2^{*}-2=\frac{4}{n-2}$, we have $\frac{\alpha n}{2}=\frac{2 n}{n-2}=2^{*}$, and thus our hypothesis reduces to supposing $u \in L^{2^{*}}$. In particular, if $f$ satisfies the growth condition in (c), every solution $u$ of (4) that belongs to the space $\mathcal{D}^{1,2}\left(\mathbb{R}^{n}\right)$ is radial.

2) We will see in Theorem 5 that in case (c) it is enough to suppose that $u \in$ $\mathcal{D}^{1, p}\left(\mathbb{R}^{n}\right)=\left\{u \in L^{p^{*}}\left(\mathbb{R}^{n}\right):|D u| \in L^{p}\left(\mathbb{R}^{n}\right)\right\}$, where $p^{*}=\frac{n p}{n-p}$, for some $p \in[2, n)$. This is relevant in particular for supercritical problems, i.e. when $\alpha>2^{*}-2=\frac{4}{n-2}$, $\frac{\alpha n}{2}>\frac{2 n}{n-2}=2^{*}$ and in general solutions of (4) do not belong to the space $\mathcal{D}^{1,2}\left(\mathbb{R}^{n}\right)$. When $f$ is as in (b) or (c), a solution $u \in L^{\alpha \frac{n}{2}}\left(\mathbb{R}^{n}\right)$ which tends to zero, respectively belongs to $\mathcal{D}^{1, p}\left(\mathbb{R}^{n}\right)$, is symmetric even if it has infinite energy, i.e. if $|D u| \notin L^{2}\left(\mathbb{R}^{n}\right)$.

In this paper, using the same type of ideas and taking advantage of a general Sobolev inequality on manifolds (see (13) and (24)), we will prove a series of symmetry results on general manifolds satisfying appropriate conditions (in particular, convenient curvature bounds).

In fact, one of the main purposes of this work is to study the very interesting relationship between the geometric properties of the manifold considered (best constants in Sobolev inequality (24) which are related to the scalar curvature of the manifold) and the analytical conditions required on the nonlinearity $f$ (growth conditions near the origin) in order to obtain our symmetry results.

For example, in the case of the standard $n$-dimensional hyperbolic space $\mathbb{H}^{n}$, we prove the following result, which is the equivalent of Theorem 1 . In this case the space 
corresponding to the space $\mathcal{D}^{1,2}\left(\mathbb{R}^{n}\right)$ is the Sobolev space $H^{1}\left(\mathbb{H}^{n}\right)$, which is the closure of $C_{c}^{\infty}\left(\mathbb{H}^{n}\right)$ with the $L^{2}$ norm of the gradient, thanks to the Sobolev inequality (13) for the hyperbolic space.

THEOREM 2. - Suppose $f$ is locally Lipschitz continuous in $(0, \infty)$ and let $u \in$ $C^{1}\left(\mathbb{H}^{n}\right), n \geqslant 3$, be a (weak) solution of

$$
\begin{cases}-\Delta_{\mathbb{H}^{n}} u=f(u) & \text { in } \mathbb{H}^{n}, \\ u>0 & \text { in } \mathbb{H}^{n} .\end{cases}
$$

Then, $u$ is radially symmetric and strictly radially decreasing around some point $x_{0} \in \mathbb{H}^{n}$ provided one of the following holds

(a) $u(x) \rightarrow 0$ as $x \rightarrow \infty$ and $\exists s_{0}>0$ such that $f$ is nonincreasing in $\left(0, s_{0}\right)$;

(b) $u(x) \rightarrow 0$ as $x \rightarrow \infty, \exists s_{0}, \alpha>0$ such that if $0<a<b<s_{0}$ then $\frac{f(b)-f(a)}{b-a} \leqslant$ $G+C(a+b)^{\alpha}$ and $u \in L^{2}\left(\mathbb{H}^{n}\right) \cap L^{\alpha \frac{n}{2}}\left(\mathbb{H}^{n}\right)$;

(c) $u \in H^{1}\left(\mathbb{H}^{n}\right), \exists \alpha>0$ such that if $0<a<b$ then $\left|\frac{f(b)-f(a)}{b-a}\right| \leqslant G+C(a+b)^{\alpha}$ and $u \in L^{\alpha \frac{n}{2}}\left(\mathbb{H}^{n}\right)$.

Here, $G=G_{\mathbb{H}^{n}}$ is the constant defined in (14) - it is a positive number which depends only on the dimension $n$.

Remark. - Suppose that $f$ satisfies the growth condition in (c) with $\alpha \in\left[\frac{4}{n}, \frac{4}{n-2}\right]$. By Sobolev inequality (13), if $u$ is a solution of (5) belonging to the closure of $C_{c}^{\infty}$ in $L^{2}$ norm of the gradient, then $u$ is radial. In a previous remark we saw that this result is also valid in the $\mathbb{R}^{n}$ setting but only for the value $\alpha=\frac{4}{n-2}$.

The paper is organized as follows.

In Section 2 we prove some symmetry results known in the case of the euclidean space, in order to introduce the technique that will be extensively used in the case of more general noncompact manifolds. In Section 3 we state some results in the case our manifold is the standard $n$-dimensional hyperbolic space $\mathbb{H}^{n}$. These are particular cases of theorems which are stated and proved in Section 4, where a class of manifolds with some symmetry and foliation properties is considered. Finally in Section 5 we show how it is possible to extend this sort of symmetry results to the case of more general manifolds.

For simplicity we assume $n \geqslant 3$ throughout the paper, although it is possible to obtain analogous results when $n=2$, using the Sobolev embeddings for this case.

\section{Some classical results in $\mathbb{R}^{n}$}

In this section we will show how our technique can be used to recover (and slightly improve) some classical symmetry results in euclidean space with a rather simple proof which can be easily extended to the case of more general manifolds. We first prove symmetry results for solutions which tend to zero at infinity. Let $u \in C^{1}\left(\mathbb{R}^{n}\right)$ be a (weak) solution of the problem

$$
\begin{cases}-\Delta u=f(u), u>0 & \text { in } \mathbb{R}^{n}, \\ u \rightarrow 0 & \text { when }|x| \rightarrow \infty,\end{cases}
$$

where we suppose that $f$ is locally Lipschitz in the open interval $(0, \infty)$. 
THEOREM 3. - Suppose there exists $s_{0}>0$ such that

(H1) $f$ in nonincreasing in $\left(0, s_{0}\right)$.

Then, $u$ is radially symmetric around some point $x_{0} \in \mathbb{R}^{n}$, i.e. $u(x)=u(r)$, where $r:=\left|x-x_{0}\right|$. Moreover, $u^{\prime}(r)<0$ for all $r>0$.

THEOREM 4. - Suppose there exist $s_{0}, \alpha>0$ such that

(H2) For all $a, b$ such that $0<a<b<s_{0}$ we have $\frac{f(b)-f(a)}{b-a} \leqslant C(a+b)^{\alpha}$ (which implies that this quotient is also $\leqslant C b^{\alpha}$ );

Then, any solution $u$ of $(6)$ that belongs to $L^{\alpha n / 2}\left(\mathbb{R}^{n}\right)$ is radially symmetric and strictly radially decreasing about some point.

The proofs of both of these theorems have an analogous structure and we will do them in parallel. As usual in radial symmetry results in $\mathbb{R}^{n}$, it suffices to fix an arbitrarily chosen direction and to prove symmetry w.r.t. that direction. We may use a system of coordinates s.t. the $x^{1}$ direction is along the direction we chose. Then, given $t \in \mathbb{R}$ we set

$$
Q_{t}:=\left\{x \in \mathbb{R}^{n}: x^{1}<t\right\} ; \quad U_{t}:=\left\{x \in \mathbb{R}^{n}: x^{1}=t\right\} \quad \text { and } \quad u_{t}(x):=u\left(x_{t}\right),
$$

where $x_{t}:=I_{t}(x):=\left(2 t-x^{1}, x^{\prime}\right)$ is the image of the point $x=\left(x^{1}, x^{\prime}\right)$ under the reflection through the hyperplane $U_{t}$. We also put

$$
Q^{t}=I_{t}\left(Q_{t}\right)
$$

In both theorems the first step of the proof will consist in showing that the set

$$
\Lambda:=\left\{t \in \mathbb{R}: \forall \mu>t, u \geqslant u_{\mu} \text { in } Q_{\mu}\right\},
$$

is nonempty and bounded from below. The second step will then be to show that if $t_{0}:=\inf \Lambda$, then $u \equiv u_{t_{0}}$ in $Q_{t_{0}}$.

\subsection{Proof of step 1: $\Lambda \neq \emptyset$ and is bounded from below}

\subsubsection{Case of Theorem 4}

First, we see that $\Lambda$ is bounded from below, since $u \rightarrow 0$ when $|x| \rightarrow \infty$.

We write $v=u_{t}$ and suppose $q \geqslant 1$ (to be chosen below). For $\varepsilon>0$, we let $w_{\varepsilon}=w_{\varepsilon, q}(x):=\left[(v-u-\varepsilon)^{+}\right]^{q}$, where ${ }^{+}$denotes the positive part of a function. Using $w_{\varepsilon}$ as test function (it has compact support in $Q_{t}$ since $u \rightarrow 0$ when $x \rightarrow \infty$ and $v \equiv u$ on $U_{t}$ ) we obtain, once we subtract the equation for $u$ from the equation for $v=u_{t}$,

$$
q \int_{Q_{t}}\left[(v-u-\varepsilon)^{+}\right]^{q-1}\left|D\left[(v-u-\varepsilon)^{+}\right]\right|^{2}=\int_{Q_{t}}[f(v)-f(u)]\left[(v-u-\varepsilon)^{+}\right]^{q} .
$$

Denote $z_{\varepsilon}:=\left[(v-u-\varepsilon)^{+}\right]^{(q+1) / 2}$. Since we integrate in a set where $v>u+\varepsilon>u>0$, if $t$ is sufficiently big $v=u_{t}<s_{0}$, and (H2) yields that, 


$$
\begin{aligned}
\frac{4 q}{(q+1)^{2}} \int_{Q_{t}}\left|D z_{\varepsilon}\right|^{2} & =\int_{Q_{t}} \frac{f(v)-f(u)}{v-u}(v-u)\left[(v-u-\varepsilon)^{+}\right]^{q} \\
& \leqslant C \int_{Q_{t}} v^{\alpha}(v-u)\left[(v-u-\varepsilon)^{+}\right]^{q} .
\end{aligned}
$$

Taking advantage of the fact that $u \in L^{\infty}\left(\mathbb{R}^{n}\right) \cap L^{\alpha n / 2}\left(\mathbb{R}^{n}\right)$, we fix a sufficiently large $q$ (say $q$ such that $\alpha+q+1 \geqslant \alpha \frac{n}{2}$ ) so that

$$
\int_{Q_{t}} v^{\alpha}(v-u)\left[(v-u-\varepsilon)^{+}\right]^{q} \leqslant \int_{Q_{t}} v^{\alpha+q+1} \leqslant \int_{\mathbb{R}^{n}} u^{\alpha+q+1}<+\infty .
$$

Passing to the limit as $\varepsilon \rightarrow 0$, and denoting $z:=z_{0}=\left[(v-u)^{+}\right]^{(q+1) / 2}$, we obtain (using the dominated convergence theorem)

$$
\int_{Q_{t}}|D z|^{2} \leqslant C \int_{Q_{t}} v^{\alpha} z^{2}
$$

Using Hölder and Sobolev inequalities it follows that

$$
\begin{aligned}
\int_{Q_{t}}|D z|^{2} & \leqslant C\left(\int_{Q_{t}} v^{\frac{\alpha n}{2}}\right)^{\frac{2}{n}}\left(\int_{Q_{t}} z^{\frac{2 n}{n-2}}\right)^{\frac{n-2}{n}} \\
& =C\left(\int_{Q^{t}=I_{t}\left(Q_{t}\right)} u^{\frac{\alpha n}{2}}\right)^{\frac{2}{n}}\left(\int_{Q_{t}} z^{2^{*}}\right)^{\frac{2}{2^{*}}} \leqslant C_{1}\left(\int_{Q^{t}} u^{\frac{\alpha n}{2}}\right)^{\frac{2}{n}} \int_{Q_{t}}|D z|^{2} .
\end{aligned}
$$

Since $u^{\alpha n / 2} \in L^{1}\left(\mathbb{R}^{n}\right)$, we have that

$$
\lim _{t \rightarrow \infty} \int_{Q^{t}} u^{\frac{\alpha n}{2}}=0
$$

and thus, for sufficiently large $t$,

$$
C_{1}\left(\int_{Q^{t}} u^{\frac{\alpha n}{2}}\right)^{\frac{2}{n}}<1
$$

Together with (9), this yields that $\int_{Q_{t}}|D z|^{2}=0$, and thus $|D z|=0$ in $Q_{t}$ and $z$ is constant in $Q_{t}$. Since $z=0$ on $U_{t}$, this implies that $z=0$ in $Q_{t}$, which proves step 1 , for Theorem 4.

\subsubsection{Case of Theorem 3}

In this case, we can simply take $q=1$ and, since $f$ is decreasing close to zero, for sufficiently big $t$ the r.h.s. in (7) is nonpositive, and therefore is zero. Passing to the limit 
as $\varepsilon \rightarrow 0$, once more we obtain

$$
\int_{Q_{t}}\left|D(v-u)^{+}\right|^{2}=0
$$

for sufficiently big $t$. We conclude as above.

\subsection{Step 2: $u \equiv u_{t_{0}}$}

Since $t_{0}$ is the infimum, by continuity we see that $u \geqslant u_{t_{0}}$ in $Q_{t_{0}}$. Thus, if we suppose $u \neq \equiv u_{t_{0}}$ in $Q_{t_{0}}$, the strong maximum principle (strong comparison principle for general operators) will yield that $u>u_{t_{0}}$ in $Q_{t_{0}}$. In fact, writing $v=u_{t_{0}}$, since $u-v=u-u_{t_{0}}$ satisfies in $Q_{t_{0}}$ the linear equation

$$
-\Delta(u-v)=c_{t_{0}}(x)(u-v), \quad \text { where } c_{t_{0}}(x)=\frac{f(u(x))-f(v(x))}{u(x)-v(x)} \in L_{\mathrm{loc}}^{\infty}\left(\mathbb{R}^{n}\right),
$$

we may apply the strong maximum principle and obtain $u>u_{t_{0}}$ in $Q_{t_{0}}$, as desired.

Since $u \in L^{\alpha n / 2}\left(\mathbb{R}^{n}\right)$, we can choose a compact $K \subset Q_{t_{0}}$ and a number $\delta>0$ such that $\forall t \in\left(t_{0}-\delta, t_{0}\right)$ we have $K \subset Q_{t}$ and

$$
C_{1}\left(\int_{I_{t}\left(Q_{t} \backslash K\right)} u^{\frac{\alpha n}{2}}\right)^{\frac{2}{n}}<\frac{1}{2}
$$

where $C_{1}$ is as in (9).

On the other hand, since $u-u_{t_{0}}$ is positive in $Q_{t_{0}}$, there exists $0<\delta_{1}<\delta$, such that

$$
u>u_{t}, \quad \text { in } K \forall t \in\left(t_{0}-\delta_{1}, t_{0}\right) .
$$

Using (10) and proceeding as in step 1, since the integrals are over $Q_{t} \backslash K$, we see that $\left(u_{t}-u\right)^{+} \equiv 0$ in $Q_{t} \backslash K$. By (11) we get $u \geqslant u_{t}$ in $Q_{t}$ for all $t \in\left(t_{0}-\delta_{1}, t_{0}\right)$, contradicting the definition of $t_{0}$.

Remarks. - (1) In the classical theorems the hypothesis is

$$
u=\mathrm{O}\left(\frac{1}{|x|^{m}}\right) \quad \text { where } \alpha m>2
$$

In that case, it follows that $u \in L^{\alpha n / 2}$ (our hypothesis) since

$$
u^{\alpha n / 2}=\mathrm{O}\left(\frac{1}{|x|^{m \alpha n / 2}}\right) \quad \text { where } m \alpha n / 2>n .
$$

(2) In the case of critical problems, i.e. when $\alpha=4 /(n-2)=2^{*}-2$, it follows that $\alpha n / 2=2 n /(n-2)=2^{*}$, and we obtain radial symmetry for solutions in $L^{2^{*}}$.

(3) Note that there could be solutions with infinite energy, i.e. whose gradient does not belong to $L^{2}$ (and this happens e.g. in the supercritical case). That is why we have to take $q \geqslant 1$ in the proof of Theorem 4 . 
If the nonlinearity $f$ satisfies the growth condition in (H2) both at zero and at infinity, then the radial symmetry of the solution $u$ follows without supposing a priori that it converges to zero at infinity, provided there exists $p$, with $2 \leqslant p<n$, such that $u \in \mathcal{D}^{1, p}\left(\mathbb{R}^{n}\right):=\left\{u \in L^{p^{*}}\left(\mathbb{R}^{n}\right):|D u| \in L^{p}\left(\mathbb{R}^{n}\right)\right\}, p^{*}=\frac{n p}{n-p}$.

More precisely we can prove the following

THEOREM 5. - Let $u \in C^{1}\left(\mathbb{R}^{n}\right)$ be a (weak) solution of

$$
\begin{cases}-\Delta u=f(u) & \text { in } \mathbb{R}^{n}, \\ u>0 & \text { in } \mathbb{R}^{n},\end{cases}
$$

where $f$ is locally Lipschitz continuous in $(0, \infty)$ and satisfies

$$
\left|\frac{f(b)-f(a)}{b-a}\right| \leqslant C(a+b)^{\alpha} \quad \text { if } 0<a<b
$$

for some $\alpha>0$. If $u \in \mathcal{D}^{1, p}\left(\mathbb{R}^{n}\right)$ for some $p \in[2, n)$, and $u \in L^{\alpha \frac{n}{2}}\left(\mathbb{R}^{n}\right)$ then $u$ is radially symmetric and strictly radially decreasing around some point $x_{0} \in \mathbb{R}^{n}$.

Proof. - Let us put $q=\frac{n(p-1)-p}{n-p}$, so that $p=\frac{n(q+1)}{n+q-1}$ and $p^{*}=(q+1) \frac{n}{n-2}$.

By the summability assumptions on the solution it is possible to take directly the function $\left[(v-u)^{+}\right]^{q}, v=u_{t}$, as a test function in the equations for $u$ and $u_{t}$ in $Q_{t}$. More precisely since $u, v$ belong to $\mathcal{D}^{1, p}\left(\mathbb{R}^{n}\right)$ and they coincide on the hyperplane $U_{t}$ there exists a sequence $\varphi_{j}$ of functions in $C_{c}^{\infty}\left(Q_{t}\right)$ such that $\varphi_{j} \rightarrow\left[(v-u)^{+}\right]$in $L^{p^{*}}\left(Q_{t}\right)$ and $D \varphi_{j} \rightarrow D(v-u)^{+}$in $L^{p}\left(Q_{t}\right)$. Moreover, passing to a subsequence and substituting if necessary $\varphi_{j}$ with $\varphi_{j}^{+}$, we can suppose that $0 \leqslant \varphi_{j} \rightarrow\left[(v-u)^{+}\right], D \varphi_{j} \rightarrow D(v-u)^{+}$a.e. in $Q_{t}$, and that there exist functions $\psi_{0} \in L^{p^{*}}, \psi_{1} \in L^{p}$, such that $\left|\varphi_{j}\right| \leqslant \psi_{0},\left|D \varphi_{j}\right| \leqslant \psi_{1}$ a.e. in $Q_{t}$.

Taking the functions $\varphi_{j}^{q}$ as test functions in the equations for $v=u_{t}$ and $u$ in $Q_{t}$ and subtracting the equations we get

$$
q \int_{Q_{t}} \varphi_{j}^{q-1} D(v-u) \cdot D \varphi_{j}=\int_{Q_{t}}[f(v)-f(u)] \varphi_{j}^{q} .
$$

If we can pass to the limit for $j \rightarrow \infty$, and obtain

$$
q \int_{Q_{t}}\left[(v-u)^{+}\right]^{q-1}\left|D\left[(v-u)^{+}\right]\right|^{2}=\int_{Q_{t}}[f(v)-f(u)]\left[(v-u)^{+}\right]^{q}<\infty,
$$

then the proof is entirely analogous to that of Theorem 4 .

So it suffices to justify the passage to the limit, which follows easily from the dominated convergence theorem. In fact we have that

$$
\left|[f(v)-f(u)] \varphi_{j}^{q}\right| \leqslant C(u+v)^{\alpha}|v-u|\left|\varphi_{j}\right|^{q} \leqslant C(u+v)^{\alpha}|v-u|\left|\psi_{0}\right|^{q}
$$

and $(u+v)^{\alpha}|v-u|\left|\psi_{0}\right|^{q}$ belongs to $L^{1}$, since $(u+v)^{\alpha} \in L^{r_{1}},|v-u| \in L^{r_{2}},\left|\psi_{0}\right|^{q} \in$ $L^{r_{3}}$, where $r_{1}=\frac{n}{2}, r_{2}=p^{*}=(q+1) \frac{n}{n-2}, r_{3}=\frac{p^{*}}{q}=\frac{q+1}{q} \frac{n}{n-2}$ with $\frac{1}{r_{1}}+\frac{1}{r_{2}}+\frac{1}{r_{3}}=1$. 
Analogously we have that

$$
\left|\varphi_{j}^{q-1} D(v-u) \cdot D \varphi_{j}\right| \leqslant\left|\psi_{0}\right|^{q-1}|D(v-u)|\left|\psi_{1}\right|
$$

and $\left|\psi_{0}\right|^{q-1}|D(v-u)|\left|\psi_{1}\right|$ belongs to $L^{1}$, since $\psi_{0}^{q-1} \in L^{s_{1}},|D(v-u)|,\left|\psi_{1}\right| \in L^{s_{2}}$, where $s_{1}=\frac{p^{*}}{q-1}=\frac{q+1}{q-1} \frac{n}{n-2}, s_{2}=p=\frac{n(q+1)}{n+q-1}$ and $\frac{1}{s_{1}}+2 \frac{1}{s_{2}}=1$.

\section{Some results in the hyperbolic space $\mathbb{H}^{n}$}

In this section we will state the main results for hyperbolic space with the standard metric. Their proofs will be an easy consequence of the results that will be stated and proved in the next section which concern manifolds on which we have a nice group action which is the case of $\mathbb{H}^{n}$.

\subsection{Relevant Sobolev inequality and main theorems}

For the $\mathbb{H}^{n}$ results we take advantage of the following Sobolev inequality (which is a special case of a more general inequality (24) we will use in the following sections). Let $u$ belong to the Sobolev space $H^{1}\left(\mathbb{H}^{n}\right)$. Then

$$
\left(\int_{\mathbb{H}^{n}}|u|^{\frac{2 n}{n-2}} \mathrm{~d} v o l\right)^{\frac{n-2}{n}} \leqslant(K(n, 2))^{2} \int_{\mathbb{H}^{n}}|D u|^{2} \mathrm{~d} v o l+B \int_{\mathbb{H}^{n}} u^{2} \mathrm{~d} v o l,
$$

where the constant $K(n, 2)$ is a universal constant that depends only on the dimension, whose exact value is $\sqrt{4 /\left(n(n-2) \omega_{n}^{2 / n}\right)}$, and $B=B_{\mathbb{H}^{n}}:=-1 / \omega_{n}^{2 / n}$ (as usual, $\omega_{n}$ denotes the volume of the standard $n$-dimensional unit sphere $\left.\left(S^{n} \subset \mathbb{R}^{n+1}\right)\right)$. We will also denote by $G=G_{\mathbb{H}^{n}}$ the ratio

$$
G=G_{\mathbb{H}^{n}}:=\frac{\left|B_{\mathbb{H}^{n}}\right|}{(K(n, 2))^{2}}=\frac{n(n-2)}{4}
$$

which plays an important role in the following results.

In the following Theorems 6-8 we assume that $u$ is a $C^{1}$ weak solution of the problem

$$
\begin{gathered}
-\Delta_{\mathbb{H}^{n}} u=f(u), \quad u>0 \quad \text { in } \mathbb{H}^{n}, \\
u \rightarrow 0 \quad \text { when } x \rightarrow \infty,
\end{gathered}
$$

where $f \in C^{1}([0,+\infty])$.

THEOREM 6. - Under the above assumptions, if there exists a $\delta>0$ s.t.

$$
f^{\prime}(s) \leqslant 0, \quad \forall s \in(0, \delta)
$$

then, $u$ is radially symmetric and strictly radially decreasing around some point $x_{0} \in \mathbb{H}^{n}$. 
THEOREM 7. - Under the above assumptions, if

$$
f^{\prime}(0)<\mu<G,
$$

where $G$ is defined in (14), and either

(a) $u \in L^{r}$ for some $r \leqslant h(\mu)$ where $h(\mu)>2$ is given by

$$
h(\mu):=\frac{2 G}{\mu}+2 \sqrt{\left(\frac{G}{\mu}\right)^{2}-\frac{G}{\mu}} ;
$$

(b) $u \in L^{2}\left(\omega^{2} \mathrm{~d} v o l\right)$, with a weight $\omega \in C^{1}\left(\mathbb{H}^{n}\right)$ s.t.

$$
\frac{|D \omega|^{2}}{\omega^{2}}<G-\mu
$$

then, $u$ is radially symmetric and strictly radially decreasing around some point $x_{0} \in \mathbb{H}^{n}$.

Remark. - In (b) we may take $\omega(x)=\exp \left(-\sqrt{a} d\left(x, y_{0}\right)\right)$, where $0<a<G-\mu$ and $y_{0}$ is any fixed point in $\mathbb{H}^{n}$.

THEOREM 8. - Under the above assumptions, if

$$
\exists \delta>0, C>0: f^{\prime}(s) \leqslant G+C s^{\alpha}, \quad \forall s \in[0, \delta],
$$

and $u \in L^{2} \cap L^{\alpha n / 2}$, then $u$ is radially symmetric and strictly radially decreasing around some point $x_{0} \in \mathbb{H}^{n}$.

Remark. - We can take $f$ locally Lipschitz in $(0,+\infty)$ instead of $C^{1}$ as we did in the statements of the theorems above. In that case, for Theorems 7 and 8 we should add the following asymptotic behavior at 0 condition

$$
\exists \delta>0, C \geqslant 0 \text { s.t. if } 0<u<v<\delta, \quad \frac{f(v)-f(u)}{v-u} \leqslant C(u+v)^{\alpha}+\mu,
$$

where

$-C=\mu=0$ in Theorem 6 ,

$-\mu<G$ in Theorem 7,

$-\mu \leqslant G$ in Theorem 8 .

Moreover, as in the case of $\mathbb{R}^{n}$, if the behavior of $f$ is known both at zero and at infinity, we can omit the condition that $u \rightarrow 0$ at $\infty$ in the hypotheses. In this spirit, we can state the following

THEOREM 9. - Let $u \in C^{1}\left(\mathbb{H}^{n}\right) \cap H^{1}\left(\mathbb{H}^{n}\right)$ be a solution of (15), where $f$ is locally Lipschitz continuous in $(0,+\infty)$ and satisfies for all $v>u>0$,

$$
\left|\frac{f(v)-f(u)}{v-u}\right| \leqslant G+C v^{\alpha},
$$

for some $C, \alpha>0$. If $u \in L^{\alpha n / 2}\left(\mathbb{H}^{n}\right)$, then $u$ is radially symmetric and strictly radially decreasing around some point $x_{0} \in \mathbb{H}^{n}$. 
Remark. - Of course if $u \in H^{1}\left(\mathbb{H}^{n}\right)$ then $u \in L^{t}\left(\mathbb{H}^{n}\right)$ for each $t \in\left[2,2^{*}\right]$, and if $\alpha \in\left[\frac{4}{n}, \frac{4}{n-2}\right]$ the condition $u \in L^{\alpha n / 2}$ is superfluous.

As we mentioned above, we will give the proofs of these theorems in a more general setting in the following section. We close this section by an example where we can apply the previous results.

\subsection{Example}

We consider hyperbolic space $\mathbb{H}^{n}$. More precisely, using stereographic coordinates, we write $(\mathcal{M}, g):=\left(B(0,1), \frac{4}{\left(1-|x|^{2}\right)^{2}} I d\right)$, where $B(0,1) \subset \mathbb{R}^{n}$ denotes the standard unit ball and $I d$ the identity matrix. In this case, the Laplace-Beltrami operator can be written as

$$
-\Delta_{g}=-\sum_{i=1}^{n} \frac{1}{4}\left(1-|x|^{2}\right)^{n} \frac{\partial}{\partial x^{i}}\left(\left(1-|x|^{2}\right)^{2-n} \frac{\partial}{\partial x^{i}}\right) .
$$

Set $u_{\alpha}(x):=\left(\frac{1-|x|^{2}}{1+|x|^{2}}\right)^{\alpha}$, where $\alpha>0$. A direct computation yields

$$
\begin{aligned}
-\Delta_{g} u_{\alpha}= & \sum_{i=1}^{n} \frac{1}{4}\left(1-|x|^{2}\right)^{n} \frac{\partial}{\partial x^{i}}\left(\left(1-|x|^{2}\right)^{2-n}\left(\frac{1-|x|^{2}}{1+|x|^{2}}\right)^{\alpha-1} \frac{4 \alpha x^{i}}{\left(1+|x|^{2}\right)^{2}}\right) \\
= & \sum_{i=1}^{n}\left(1-|x|^{2}\right)^{n} \frac{\partial}{\partial x^{i}}\left(\frac{\alpha\left(1-|x|^{2}\right)^{1-n+\alpha} x^{i}}{\left(1+|x|^{2}\right)^{\alpha+1}}\right) \\
= & \alpha n\left(\frac{1-|x|^{2}}{1+|x|^{2}}\right)^{\alpha+1}+\sum_{i=1}^{n} \alpha x^{i}\left(1-|x|^{2}\right)^{n} \frac{\partial}{\partial x^{i}}\left(\frac{\left(1-|x|^{2}\right)^{1-n+\alpha}}{\left(1+|x|^{2}\right)^{\alpha+1}}\right) \\
= & \frac{\left(1-|x|^{2}\right)^{\alpha}}{\left(1+|x|^{2}\right)^{\alpha+2}}\left[\alpha n\left(1-|x|^{4}\right)-2 \alpha(1-n+\alpha)\left(|x|^{2}+|x|^{4}\right)\right. \\
& \left.-2 \alpha(\alpha+1)\left(|x|^{2}-|x|^{4}\right)\right] \\
= & \alpha(n-1-\alpha)\left(\frac{1-|x|^{2}}{1+|x|^{2}}\right)^{\alpha}+\alpha(\alpha+1)\left(\frac{1-|x|^{2}}{1+|x|^{2}}\right)^{\alpha+2} \\
= & \alpha(n-1-\alpha) u_{\alpha}+\alpha(\alpha+1) u_{\alpha}^{\frac{\alpha+2}{\alpha}}=: f_{\alpha}\left(u_{\alpha}\right) .
\end{aligned}
$$

We remark that for all $\alpha>0 u_{\alpha} \rightarrow 0$ when $|x|=|x|_{\mathbb{R}^{n}} \rightarrow 1$, i.e. at the boundary of the ball (which corresponds to $\infty$ of $\mathbb{H}^{n}$ ).

We consider general solutions of this equation, i.e. solutions $v_{\alpha}$ of

$$
\left\{\begin{array}{l}
-\Delta_{g} v_{\alpha}=f_{\alpha}\left(v_{\alpha}\right)=\alpha(n-1-\alpha) v_{\alpha}+\alpha(\alpha+1) v_{\alpha}^{\frac{\alpha+2}{\alpha}} \\
v_{\alpha} \rightarrow 0 \text { when } x \rightarrow \partial B(0,1), \quad v_{\alpha}>0 \text { in } B(0,1) .
\end{array}\right.
$$

We discuss the following interesting cases

(1) If $\alpha>n-1$, then $f_{\alpha}^{\prime}(0)<0$. It follows from Theorem 6 that any positive solution $v_{\alpha}$ of (23) is radially symmetric.

(2) If $n / 2<\alpha \leqslant n-1$, then $f_{\alpha}^{\prime}(0)<G$. In this case, the radial symmetry of any $v_{\alpha} \in L^{2}\left(\mathbb{H}^{n}\right)$ follows from Theorem 7 . 
(3) If $\alpha=n / 2$, we have the limit case. We notice that $\forall v>u>0$

$$
\left|\frac{f(v)-f(u)}{v-u}\right| \leqslant G+\left(1+\frac{4}{n}\right) v^{\frac{4}{n}}
$$

Moreover $\frac{n}{2} \times \frac{4}{n}=2$, so by Theorem 9 if $v_{n / 2} \in H^{1}\left(\mathbb{H}^{n}\right)$ then it is radially symmetric.

\section{Manifolds with "group symmetries"}

We will start by studying a special class of manifolds having nice symmetry properties and where the less geometry-oriented reader should still feel quite comfortable - the group action properties we assume in this case make things very similar to what happens in the familiar case $\mathbb{R}^{n}$ (and $\mathbb{H}^{n}$, which is a particular element of this class of manifolds). In Section 5 we will work under more general conditions.

\subsection{Assumptions on the manifold and Sobolev inequality}

In this section and the following one we will consider complete Riemannian nmanifolds $(\mathcal{M}, g)$ satisfying certain foliation conditions and appropriate bounds on the Riemann curvature tensor. We first indicate the general conditions needed on the manifold to have the Sobolev inequality we will use in both Sections 4 and 5 (a generalized version of (13)).

Both here and in Section 5, we assume the following bounds on $R$, the Riemann curvature tensor of $(\mathcal{M}, g)$,

$$
\exists \Lambda_{1}, \Lambda_{2} \geqslant 0: \quad|R| \leqslant \Lambda_{1} \quad \text { and } \quad|\nabla R| \leqslant \Lambda_{2},
$$

where $\nabla$ denotes the covariant derivative. Moreover, we suppose that the injectivity radius is strictly positive, i.e.

$$
\operatorname{inj}(\mathcal{M}, g)=\rho>0 .
$$

Then, by Theorem 4.12 of Hebey [13], we have the following Sobolev inequality for functions in the Sobolev space $H^{1}\left(\mathbb{H}^{n}\right)$ :

$$
\left(\int_{\mathcal{M}}|v|^{\frac{2 n}{n-2}} \mathrm{~d} v o l\right)^{\frac{n-2}{n}} \leqslant(K(n, 2))^{2} \int_{\mathcal{M}}|D v|^{2} \mathrm{~d} v o l+B \int_{\mathcal{M}} v^{2} \mathrm{~d} v o l,
$$

where $K(n, 2)$ is a universal constant depending only on the dimension $n$ and $B=B_{\mathcal{M}}$ is a constant depending on the manifold. The exact value of $K$ is

$$
K(n, 2)=\frac{1}{n} \sqrt{\frac{n}{n-2}}\left(\frac{\Gamma(n+1)}{\Gamma\left(\frac{n}{2}\right) \Gamma\left(\frac{n}{2}+1\right) \omega_{n-1}}\right)^{1 / n}=\sqrt{\frac{4}{n(n-2) \omega_{n}^{2 / n}}}
$$


(see [13], pp. 61, 69) where $\omega_{n-1}$ denotes the volume of the standard $(n-1)$-dimensional unit sphere $\left(S^{n-1} \subset \mathbb{R}^{n}\right)$. As in the case of $\mathbb{H}^{n}$, the constant

$$
G=G_{\mathcal{M}}:=\frac{\left|B_{\mathcal{M}}\right|}{(K(n, 2))^{2}}
$$

plays a crucial role in the analysis.

In this section we will make one further important assumption about our manifold: we suppose that

$$
B_{\mathcal{M}}<0 \text {. }
$$

This hypothesis is in particular satisfied when $\mathcal{M}=\mathbb{H}^{n}$, the standard hyperbolic space, in which case $B=B_{\mathbb{H}^{n}}:=-1 / \omega_{n}^{2 / n}$ (see [13], p. 81).

\subsection{The "group symmetry" foliation properties}

We will indicate the nice symmetry and foliation properties of $(\mathcal{M}, g)$ which we will assume in this section. They are of the same type as those in [2] - they may seem rather technical at first sight, but they are just the natural generalization of the well-known reflection with respect to hyperplanes and foliation by hyperplanes for $\mathbb{R}^{n}$.

We assume that there is a family of isometries $A_{t}$ (which generalize translations, or rotations, in the $\mathbb{R}^{n}$ case) and a reflection $I$ (i.e. an isometry such that $I^{2}=I d$, and $I$ fixes a hypersurface $U \subset \mathcal{M}$ ) s.t. we have the following invariance condition

$$
\forall t \in \mathbb{R}, \quad A_{t} I A_{t}=I .
$$

We suppose $A_{t}$ is a one-parameter group of transformations which is $C^{1}(\mathbb{R} \times \mathcal{M}, \mathcal{M})$, and define the vector field

$$
X(y):=\left.\frac{\partial A_{t}(y)}{\partial t}\right|_{t=0} \quad \text { for } y \in \mathcal{M} .
$$

Then, $A_{t}=\exp (t X)$.

We can "translate" the reflection $I$ using $A_{t}$ to define a one-parameter family of reflections

$$
I_{t}=A_{t} I A_{-t} .
$$

Let $U_{t}$ be the hypersurface of $\mathcal{M}$ which is fixed by $I_{t}$. We assume that $\mathcal{M}$ can be foliated by the $U_{t}$ : the $U_{t}$ should be pairwise disjoint, $\forall t_{1}, t_{2} \in \mathbb{R}, \bigcup_{t_{1}<t<t_{2}} U_{t}$ should be an open subset of $\mathcal{M}$, and $\mathcal{M}=\bigcup_{t \in \mathbb{R}} U_{t}$.

We notice the following very useful relation between the group of transformations $A_{t}$ and the family of isometries $I_{t}$, which motivated calling (27) an invariance condition,

$$
\forall t, \tau \in \mathbb{R}, \forall x \in U_{t}, \quad I_{\tau}(x)=A_{2(\tau-t)}(x) .
$$

This implies, in particular, that if $\Sigma(x)=\left\{A_{t}(x): t \in \mathbb{R}\right\}$, is the trajectory of any $x \in \mathcal{M}$ under the action of the transformation group $A_{t}$ (which is the same as the integral trajectory of $x$ under the flow of $X)$, then $\forall \tau \in \mathbb{R}, I_{\tau}(\Sigma(x))=\Sigma(x)$. 
The proof of (29) is very simple. In fact, since $x \in U_{t}, I_{t}(x)=x$, and thus

$$
\begin{aligned}
I_{\tau}(x) & =A_{\tau} I A_{-\tau} I_{t}(x)=A_{\tau} I A_{-\tau} A_{t} I A_{-t}(x)=A_{\tau} I A_{t-\tau} I A_{-t}(x) \\
& =A_{\tau} I A_{t-\tau} I A_{t-\tau} A_{\tau-2 t}(x)=A_{\tau} I^{2} A_{\tau-2 t}(x)=A_{2(\tau-t)}(x) .
\end{aligned}
$$

For $t_{1} \in \mathbb{R}$ we define

$$
Q_{t_{1}}=\bigcup_{-\infty<t<t_{1}} U_{t} \text { and } Q^{t_{1}}=\bigcup_{t_{1}<t<+\infty} U_{t}
$$

We should have $I_{t}\left(Q_{t}\right) \subset Q^{t}$ and $I_{t}\left(Q^{t}\right) \subset Q_{t}$, for all $t \in \mathbb{R}$. For $t \in \mathbb{R}$ and $x \in Q_{t}$ we define $x_{t}=I_{t}(x)$ and $u_{t}(x)=u\left(x_{t}\right)$. The function $t(x)$ assigns to each $x \in \mathcal{M}$ the unique $t \in \mathbb{R}$ s.t. $x \in U_{t}$. It is a continuous function on $\mathcal{M}$.

We will start by showing that our assumptions imply that the action of the group is orthogonal to the invariant hypersurfaces and that along the integral trajectories of $X$ we exit any compact subset of $\mathcal{M}$ in finite time.

Lemma 1. - Let $x \in \mathcal{M}$ be an arbitrary point and $\tau=t(x)$ (so that $x \in U_{\tau}$ ). Then, $\left.\frac{\partial A_{t}(x)}{\partial t}\right|_{t=0} \neq 0$, and is orthogonal to $T_{x} U_{\tau}$. Thus

$$
\left.T_{x} U_{\tau} \oplus \frac{\partial A_{t}(x)}{\partial t}\right|_{t=0}=T_{x} \mathcal{M}
$$

Proof. - Since $A_{t}$ is a group and $I_{t}=A_{t} I A_{-t}$, to prove the lemma it suffices to show that for all $x \in U,\left.\frac{\partial A_{t}(x)}{\partial t}\right|_{t=0} \neq 0$ and is orthogonal to $U$ (we can then translate this result using $A_{t}$ ).

For $x \in U$, using the invariance condition we have

$$
A_{t} I A_{t}(x)=I(x)=x \Leftrightarrow A_{t}(x)=I A_{-t} I(x)=I A_{-t}(x) .
$$

Differentiating this relationship at $x \in U$, we obtain

$$
\left.\frac{\partial A_{t}(x)}{\partial t}\right|_{t=0}=\mathrm{d} I\left(\left.\frac{\partial A_{-t}(x)}{\partial t}\right|_{t=0}\right)=\mathrm{d} I\left(-\left.\frac{\partial A_{t}(x)}{\partial t}\right|_{t=0}\right) .
$$

Since $I$ is an isometry and $X(x)=\left.\frac{\partial A_{t}(x)}{\partial t}\right|_{t=0}$ satisfies, according to the relation above, $X(x)=\mathrm{d} I(-X(x))$, it follows that $X$ is orthogonal to the hypersurface fixed by $I$, i.e. $X \perp U$.

If we had $X(x)=0$, then we would have $A_{t}(x)=\exp (t X(x))=x, \forall t \in \mathbb{R}$. However, since $A_{t}(x) \in U_{t}$ this would imply that $x \in U_{t} \forall t \in \mathbb{R}$, contradicting our assumption that the $U_{t}$ are pairwise disjoint.

LEMMA 2. - Given any point $x \in \mathcal{M}$, and any compact subset $K \subset \mathcal{M}$ containing $x$, then there exists $\tau=\tau(x, K)<+\infty$ s.t. integral trajectory $A_{t}(x)=\exp (t X)(x)$ does not belong to $K$ for $|t|>\tau$ (i.e. the trajectory permanently exits $K$ in finite positive and negative $t$ ).

Proof. - This lemma is just an immediate consequence of the continuity of $t$. In fact, since $t$ is continuous and $K$ is compact, $t(K)$ is a compact subset of $\mathbb{R}$, call it $K_{1}$. 
Since, from the group property of $A_{t}$ and the definition of the function $t$, we have $t\left(A_{s}(x)\right)=s+t(x)$, our conclusion follows from the fact that $K_{1}$ is bounded.

As an example, we can consider foliations of hyperbolic space $\mathbb{H}^{n}$ (see [2]).

Let $\mathbb{R}^{n, 1}=\left(\mathbb{R}^{n+1}, g\right)$ be the Minkowski space, where $g$ is the metric with signature $(-,+, \ldots,+)$. Hyperbolic space of dimension $n, \mathbb{H}^{n}$, is the submanifold $\{x \in$ $\mathbb{R}^{n, 1}: g(x, x)=-1$, and $\left.x^{0}>0\right\}$.

A particular directional foliation can be obtained by choosing any direction in the $x^{1}, \ldots, x^{n}$ plane. For simplicity let us suppose that the direction considered is the $x^{1}$ direction. We may then write $\mathbb{R}^{n, 1}=\mathbb{R}^{1,1} \times \mathbb{R}^{n-1}$, and define $A_{t}=\tilde{A}_{t} \otimes I d_{\mathbb{R}^{n-1}}$, where $\tilde{A}_{t}$ is the hyperbolic rotation of angle $t$ in $\mathbb{R}^{1,1}$, i.e.

$$
\tilde{A}_{t}=\left(\begin{array}{cc}
\cosh (t) & \sinh (t) \\
\sinh (t) & \cosh (t)
\end{array}\right): \mathbb{R}^{1,1} \rightarrow \mathbb{R}^{1,1}
$$

The reflection $I$ is defined to be $\left(x^{0}, x^{1}, x^{2}, \ldots, x^{n}\right) \mapsto\left(x^{0},-x^{1}, x^{2}, \ldots, x^{n}\right)$.

Then, $U=\mathbb{H}^{n} \cap\left\{x^{1}=0\right\}$ and $U_{t}=A_{t}(U)$ for all $t \in \mathbb{R}$. Moreover, $\mathbb{H}^{n}$ is foliated by $U_{t}$.

We will state and prove the equivalents of Theorems 6-9 in the general setting of this section, and we will give their proofs. As we will show in Section 4.7, the original radial symmetry results in $\mathbb{H}^{n}$ of Theorems 6-9 are then easy corollaries of their general versions. In the following Theorems 10-12 we suppose that $B_{\mathcal{M}}<0$ and that $u$ is a $C^{1}$ weak solution of

$$
\begin{gathered}
-\Delta_{\mathcal{M}} u=f(u), u>0 \quad \text { in } \mathcal{M}, \\
u \rightarrow 0 \quad \text { when } x \rightarrow \infty,
\end{gathered}
$$

where $f \in C^{1}([0,+\infty])$. In (31) we mean that if we fix any point $x_{1} \in \mathcal{M}$, then $u(x) \rightarrow 0$ when the distance from $x$ to $x_{1}$ tends to $+\infty$.

Fixing a group action $A_{t}$ and a reflection $I$ (and the associated foliation $U_{t}$ ) means that we choose some direction to move and reflect things. Our theorems will state sufficient conditions for solutions $u$ of (30) and (31) to be symmetric along this direction: existence of a $\bar{\lambda} \in \mathbb{R}$ such that $u(x)$ coincides with its reflection (by $I_{\bar{\lambda}}$ ) with respect to $U_{\bar{\lambda}}$, which we called $u_{\bar{\lambda}}(x)=u\left(I_{\bar{\lambda}}(x)\right)$.

\subsection{Statements of the main theorems}

THEOREM 10. - Under the above assumptions, if there exists a $\delta>0$ s.t.

$$
f^{\prime}(s) \leqslant 0, \quad \forall s \in(0, \delta)
$$

then, $u$ is symmetric along our direction, i.e. $\exists \bar{\lambda}$ s.t. $u(x)=u_{\bar{\lambda}}(x)$.

We choose to give the proof of this result in the more general setting of Section 5 in order to illustrate how to work in that setting. Theorem 10 is an easy corollary of Theorem 14 which we will prove in Section 5. 
THEOREM 11. - Under the above assumptions, if

$$
f^{\prime}(0)<\mu<G,
$$

where $G$ is defined in (26), and either

(a) $u \in L^{r}$ for

$$
r=h(\mu):=\frac{2 G}{\mu}+2 \sqrt{\left(\frac{G}{\mu}\right)^{2}-\frac{G}{\mu}} \quad(>2) ;
$$

(b) $u \in L^{2}\left(\omega^{2} \mathrm{~d} v o l\right)$, with a weight $\omega \in C^{1}(\mathcal{M})$ s.t.

$$
\frac{|D \omega|^{2}}{\omega^{2}}<G-\mu
$$

then, $u$ is symmetric along our direction, i.e. $\exists \bar{\lambda}$ s.t. $u(x)=u_{\bar{\lambda}}(x)$.

Remark. - Since $u \in L^{\infty}\left(\mathbb{R}^{n}\right)$ by hypothesis, if $u \in L^{r}$ then $u \in L^{s}$ for all $s \geqslant r$. Therefore by (a) $u$ is symmetric provided $u \in L^{r}\left(\mathbb{R}^{n}\right)$ for some $r<r_{0}$, where $r_{0}=$ $h\left(f^{\prime}(0)\right)$.

THEOREM 12. - Under the above assumptions, if

$$
\exists \delta>0, C>0: f^{\prime}(s) \leqslant G+C s^{\alpha}, \quad \forall s \in[0, \delta],
$$

and $u \in L^{2} \cap L^{\alpha n / 2}$, then $u$ is symmetric along our direction.

In the case when the behavior of $f$ is known at zero and at infinity, we have the following result, where condition (31) is not a priori supposed.

THEOREM 13. - Let $u \in C^{1}(\mathcal{M}) \cap H^{1}(\mathcal{M})$ be a solution of (30), with $f$ locally Lipschitz continuous in $(0,+\infty)$ and satisfying for all $v>u>0$

$$
\left|\frac{f(v)-f(u)}{v-u}\right| \leqslant G+C v^{\alpha},
$$

for some $C, \alpha>0$. If $u \in L^{\alpha n / 2}$, then $u$ is symmetric along the direction considered.

Remark. - (1) It follows from the Sobolev inequality that if $v \in H^{1}(\mathcal{M})$, then $v \in$ $L^{\beta}(\mathcal{M}), \forall \beta \in\left[2, \frac{2 n}{n-2}\right]$.

(2) In Theorem $10, B_{\mathcal{M}}<0$ is unnecessary.

\subsection{Proof of Theorem 11}

\subsubsection{Statement (a)}

From (33) and the fact that we supposed $f \in C^{1}([0, \infty))$ it follows that there exists a $\delta>0$ s.t. for all $u, v \in(0, \delta]$,

$$
\frac{f(u)-f(v)}{u-v} \leqslant \mu<G .
$$


As a matter of fact, this is the good condition on $f$ for this proof.

Set $\Lambda=\left\{t \in \mathbb{R}: \forall \tau>t, u \geqslant u_{\tau}\right.$ in $\left.Q_{\tau}\right\}$. The proof consists of 3 steps.

Step 1: $\Lambda$ is nonempty.

First, we remark that, for $\lambda \in \mathbb{R}$,

$$
-\Delta_{\mathcal{M}}\left(u_{\lambda}-u\right)=f\left(u_{\lambda}\right)-f(u)
$$

Let

$$
q=h(\mu)-1=\frac{2 G}{\mu}+2 \sqrt{\left(\frac{G}{\mu}\right)^{2}-\frac{G}{\mu}}-1
$$

and fix $\varepsilon>0$. We see that $\left[\left(u_{\lambda}-u-\varepsilon\right)^{+}\right]^{q}$ has compact support since $\lim _{x \rightarrow \infty} u(x)=0$ and $u \equiv u_{\lambda}$ on $U_{\lambda}$. Taking $\left[\left(u_{\lambda}-u-\varepsilon\right)^{+}\right]^{q}$ as test function, we obtain

$$
\int_{Q_{\lambda}}-\Delta_{\mathcal{M}}\left(u_{\lambda}-u\right)\left[\left(u_{\lambda}-u-\varepsilon\right)^{+}\right]^{q} \mathrm{~d} v o l=\int_{Q_{\lambda}}\left(f\left(u_{\lambda}\right)-f(u)\right)\left[\left(u_{\lambda}-u-\varepsilon\right)^{+}\right]^{q} \mathrm{~d} v o l .
$$

Since $\lim _{x \rightarrow \infty} u(x)=0$, there exist $x_{0} \in \mathcal{M}$ and $R>0$ s.t.

$$
\forall y \in \mathcal{M} \backslash B\left(x_{0}, R\right), \quad 0<u(y)<\delta .
$$

Moreover, since $\bar{B}\left(x_{0}, R\right)$ is compact, there exists $\lambda_{0}$ s.t.

$$
B\left(x_{0}, R\right) \subset Q_{\lambda_{0}}
$$

Choosing $\lambda>\lambda_{0}$, it follows that $u_{\lambda}<\delta$ in $Q_{\lambda}$. But, since the integrand in the r.h.s. of (40) is nonzero only if $u<u_{\lambda}-\varepsilon$, we have both $u, u_{\lambda}<\delta$ in the significant integration domain and thus, using (33), it follows that

$$
\int_{Q_{\lambda}}\left(f\left(u_{\lambda}\right)-f(u)\right)\left[\left(u_{\lambda}-u-\varepsilon\right)^{+}\right]^{q} \mathrm{~d} v o l \leqslant \mu \int_{Q_{\lambda}}\left(u_{\lambda}-u\right)\left[\left(u_{\lambda}-u-\varepsilon\right)^{+}\right]^{q} \mathrm{~d} v o l .
$$

On the other hand,

$$
\begin{aligned}
\int_{Q_{\lambda}} & -\Delta_{\mathcal{M}}\left(u_{\lambda}-u\right)\left[\left(u_{\lambda}-u-\varepsilon\right)^{+}\right]^{q} \mathrm{~d} v o l \\
& =\int_{Q_{\lambda}}\left\langle D\left(u_{\lambda}-u\right), D\left[\left(u_{\lambda}-u-\varepsilon\right)^{+}\right]^{q}\right\rangle \mathrm{d} v o l \\
& =q \int_{Q_{\lambda}}\left[\left(u_{\lambda}-u-\varepsilon\right)^{+}\right]^{q-1}\left\langle D\left(u_{\lambda}-u\right), D\left(u_{\lambda}-u\right)\right\rangle \mathrm{d} v o l \\
& =\frac{4 q}{(q+1)^{2}} \int_{Q_{\lambda}}\left|D\left[\left(u_{\lambda}-u-\varepsilon\right)^{+}\right]^{\frac{q+1}{2}}\right|^{2} \mathrm{~d} v o l .
\end{aligned}
$$


Therefore, using (42), we obtain

$$
\begin{aligned}
& \frac{4 q}{(q+1)^{2}} \int_{Q_{\lambda}}\left|D\left[\left(u_{\lambda}-u-\varepsilon\right)^{+}\right]^{\frac{q+1}{2}}\right|^{2} \mathrm{~d} v o l \\
& \leqslant \mu \int_{Q_{\lambda}}\left(u_{\lambda}-u\right)\left[\left(u_{\lambda}-u-\varepsilon\right)^{+}\right]^{q} \mathrm{~d} v o l \leqslant \mu \int_{Q_{\lambda}}\left[\left(u_{\lambda}-u\right)^{+}\right]^{q+1} \mathrm{~d} v o l .
\end{aligned}
$$

Noticing that $\left[\left(u_{\lambda}-u\right)^{+}\right]^{q+1} \in L^{1}\left(Q_{\lambda}\right)$ and letting $\varepsilon \rightarrow 0$, it follows from the monotone convergence theorem that

$$
\frac{4 q}{(q+1)^{2}} \int_{Q_{\lambda}}\left|D\left[\left(u_{\lambda}-u\right)^{+}\right]^{\frac{q+1}{2}}\right|^{2} \mathrm{~d} v o l \leqslant \mu \int_{Q_{\lambda}}\left(\left[\left(u_{\lambda}-u\right)^{+}\right]^{\frac{q+1}{2}}\right)^{2} \mathrm{~d} v o l .
$$

Thus $\left[\left(u_{\lambda}-u\right)^{+}\right]^{\frac{q+1}{2}} \in H^{1}\left(Q_{\lambda}\right)$. Using the Sobolev inequality (24) we see that

$$
\begin{aligned}
& \frac{4 q}{(q+1)^{2}} \int_{Q_{\lambda}}\left|D\left[\left(u_{\lambda}-u\right)^{+}\right]^{\frac{q+1}{2}}\right|^{2} \mathrm{~d} v o l \\
& \leqslant \mu\left(\frac{K^{2}}{-B} \int_{Q_{\lambda}}\left|D\left[\left(u_{\lambda}-u\right)^{+}\right]^{\frac{q+1}{2}}\right|^{2}-\frac{1}{-B}\left(\int_{Q_{\lambda}}\left(\left[\left(u_{\lambda}-u\right)^{+}\right]^{\frac{q+1}{2}}\right)^{p} \mathrm{~d} v o l\right)^{\frac{2}{p}}\right) \\
& \left.\quad \frac{\mu K^{2}}{|B|} \int_{Q_{\lambda}}\left|D\left[\left(u_{\lambda}-u\right)^{+}\right]^{\frac{q+1}{2}}\right|^{2}-\frac{\mu}{|B|}\left(\int_{Q_{\lambda}}\left(\left[\left(u_{\lambda}-u\right)^{+}\right]^{\frac{q+1}{2}}\right)^{p} \mathrm{~d} v o l\right)^{\frac{2}{p}}\right),
\end{aligned}
$$

where $p=\frac{2 n}{n-2}$. Here, and in the conclusion of this step, it is crucial to use the fact that $B<0$ so that $-B=|B|>0$. On the other hand, it is easy to check that

$$
\frac{4 q}{(q+1)^{2}}=\frac{\mu K^{2}}{|B|}=\frac{\mu}{G}>0
$$

and thus it follows that

$$
\int_{Q_{\lambda}}\left(\left[\left(u_{\lambda}-u\right)^{+}\right]^{\frac{q+1}{2}}\right)^{p} \mathrm{~d} v o l \leqslant 0 .
$$

Thus $\left(u_{\lambda}-u\right)^{+} \equiv 0$ in $Q_{\lambda}$, which means that $u_{\lambda} \leqslant u$ in $Q_{\lambda}$ and, consequently, $\lambda \in \Lambda$.

Step 2: $\Lambda$ is bounded below.

By assumption (31)

$$
\lim _{\lambda \rightarrow-\infty} \sup _{Q_{\lambda}} u=0,
$$

and thus, we may choose some $\sigma_{1}$ s.t. $\sup _{Q_{\sigma_{1}}} u<\left(\sup _{\mathcal{M}} u\right) / 2$. This implies that all $\lambda \in\left(-\infty, \sigma_{1}\right)$ do not belong to $\Lambda$. Therefore, $\Lambda$ is bounded from below, and we let

$$
\bar{\lambda}:=\inf \Lambda \text {. }
$$


Step 3: $u \equiv u_{\bar{\lambda}}$ in $Q_{\bar{\lambda}}$.

In fact, it is clear that by continuity of the foliation and of $u$, we have $u \geqslant u_{\bar{\lambda}}$ in $Q_{\bar{\lambda}}$.

Using our equation and the assumption that $f$ is locally Lipschitz, we have that $\forall x \in Q_{\bar{\lambda}}, \exists M, r>0$ s.t. for all $y \in B(x, r) \cap Q_{\bar{\lambda}}$, we have

$$
-\Delta_{\mathcal{M}}\left(u-u_{\bar{\lambda}}\right)=f(u)-f\left(u_{\bar{\lambda}}\right) \geqslant-M\left(u-u_{\bar{\lambda}}\right) .
$$

Thus, the strong maximum principle and connectedness of $Q_{\bar{\lambda}}$ imply that either $u \equiv u_{\bar{\lambda}}$ in $Q_{\bar{\lambda}}$ (in which case the proof of Theorem 11 is complete), or else $u>u_{\bar{\lambda}}$ in $Q_{\bar{\lambda}}$.

Suppose the latter case were true. Choose $x_{1} \in U_{\bar{\lambda}}$ and $R^{\prime}>0$ s.t. $B\left(x_{0}, R\right) \subset$ $B\left(x_{1}, R^{\prime}\right)$. By the continuity of the foliation, there would exist $\eta_{0}>0$ s.t. for $0<$ $\eta<\eta_{0}, \quad I_{\bar{\lambda}-\eta}\left(B\left(x_{1}, R^{\prime}\right)\right) \subset B\left(x_{1}, 2 R^{\prime}\right)$. Moreover, by the definition of $\bar{\lambda}$ we could construct an increasing sequence $\lambda_{n} \nearrow \bar{\lambda}$ such that $\lambda_{n}>\bar{\lambda}-\eta_{0}$ and $\exists y_{n} \in Q_{\lambda_{n}}$ s.t. $u\left(y_{n}\right)<u\left(I_{\lambda_{n}}\left(y_{n}\right)\right)=u_{\lambda_{n}}\left(y_{n}\right)$.

We claim that $y_{n} \in B\left(x_{1}, 2 R^{\prime}\right)$. If this were not so, taking $\left(u_{\lambda_{n}}-u-\varepsilon\right)^{+}$as test function (as in the previous steps), and using the fact that $0<u \leqslant u_{\lambda_{n}}<\delta$ in $\operatorname{supp}\left(u_{\lambda_{n}}-u-\varepsilon\right)^{+}$, we would conclude that $u_{\lambda_{n}} \leqslant u$ in $Q_{\lambda_{n}}$. This proves our claim.

Modulo a subsequence, there would exist $y \in Q_{\bar{\lambda}}$, such that $y_{n} \rightarrow y$. By continuity, we should have

$$
u(y)=\lim _{n \rightarrow+\infty} u\left(y_{n}\right) \leqslant \lim _{n \rightarrow+\infty} u_{\lambda_{n}}\left(y_{n}\right)=u_{\bar{\lambda}}(y) .
$$

This would imply that $y \in U_{\bar{\lambda}}$. On the other hand, there would exist points $\zeta_{n}$ in the line segment between $y_{n}$ and $I_{\lambda_{n}}\left(y_{n}\right)$ s.t. $X(u)\left(\zeta_{n}\right) \leqslant 0$, where $X$ is, as before, the Killing vector field associated to the transformation group $A_{t}$. Passing to the limit we should have $X(u)(y) \leqslant 0$. However, this is impossible since by the strong maximum principle $X(u)(x)>0$ for all $x \in U_{\bar{\lambda}}$. This contradiction implies that, as desired, $u \equiv u_{\bar{\lambda}}$ in $Q_{\bar{\lambda}}$.

\subsubsection{Statement (b)}

As before, set $\Lambda=\left\{t \in \mathbb{R}: \forall \tau>t, u \geqslant u_{\tau}\right.$ in $\left.Q_{\tau}\right\}$. The proof consists of 3 steps, but we only need to give the proof of step 1 since the other two are analogous to those given in case (a).

Step 1: $\Lambda$ is nonempty.

As above, we define $\lambda_{0}$ (see (41)) and take $\lambda>\lambda_{0}$. For any $\varepsilon>0$ we take $\left(u_{\lambda}-u-\right.$ $\varepsilon)^{+} \omega^{2}$ as test function. A direct computation yields

$$
\begin{aligned}
\int_{Q_{\lambda}} & -\Delta_{\mathcal{M}}\left(u_{\lambda}-u\right)\left(u_{\lambda}-u-\varepsilon\right)^{+} \omega^{2} \mathrm{~d} v o l \\
& =\int_{Q_{\lambda}}\left(f\left(u_{\lambda}\right)-f(u)\right)\left(u_{\lambda}-u-\varepsilon\right)^{+} \omega^{2} \mathrm{~d} v o l \\
& \leqslant \mu \int_{Q_{\lambda}}\left(u_{\lambda}-u\right)\left(u_{\lambda}-u-\varepsilon\right)^{+} \omega^{2} \mathrm{~d} v o l \leqslant \mu \int_{Q_{\lambda}}\left[\left(u_{\lambda}-u\right)^{+}\right]^{2} \omega^{2} \mathrm{~d} v o l .
\end{aligned}
$$


On the other hand,

$$
\begin{aligned}
\int_{Q_{\lambda}} & -\Delta_{\mathcal{M}}\left(u_{\lambda}-u\right)\left(u_{\lambda}-u-\varepsilon\right)^{+} \omega^{2} \mathrm{~d} v o l \\
= & \int_{Q_{\lambda}}\left\langle D\left(u_{\lambda}-u\right), D\left(u_{\lambda}-u-\varepsilon\right)^{+} \omega^{2}\right\rangle \mathrm{d} v o l \\
= & \int_{Q_{\lambda} \cap\left\{u_{\lambda}>u+\varepsilon\right\}}\left\langle D\left(u_{\lambda}-u-\varepsilon\right)^{+}, D\left(u_{\lambda}-u-\varepsilon\right)^{+} \omega^{2}\right\rangle \mathrm{d} v o l \\
= & \int_{Q_{\lambda}}\left\langle D\left(\frac{Z_{\varepsilon}}{\omega}\right), D\left(Z_{\varepsilon} \omega\right)\right\rangle \mathrm{d} v o l,
\end{aligned}
$$

where $Z_{\varepsilon}:=\omega\left(u_{\lambda}-u-\varepsilon\right)^{+}$. Continuing the computation, we see that the last integral is

$$
\begin{aligned}
\int_{Q_{\lambda}} & \left\langle D\left(\frac{Z_{\varepsilon}}{\omega}\right), \omega D\left(Z_{\varepsilon}\right)\right\rangle+\left\langle D\left(\frac{Z_{\varepsilon}}{\omega}\right), Z_{\varepsilon} D(\omega)\right\rangle \mathrm{d} v o l \\
= & \int_{Q_{\lambda}}\left|D Z_{\varepsilon}\right|^{2}-\left\langle\frac{Z_{\varepsilon}}{\omega} D \omega, D\left(Z_{\varepsilon}\right)\right\rangle+\left\langle D\left(\frac{Z_{\varepsilon}}{\omega}\right), Z_{\varepsilon} D(\omega)\right\rangle \mathrm{d} v o l \\
= & \int_{Q_{\lambda}}\left|D Z_{\varepsilon}\right|^{2}-\left\langle\frac{Z_{\varepsilon}}{\omega} D \omega, D\left(Z_{\varepsilon}\right)\right\rangle+\left\langle\frac{D Z_{\varepsilon}}{\omega}, Z_{\varepsilon} D(\omega)\right\rangle+Z_{\varepsilon}^{2}\left\langle D\left(\frac{1}{\omega}\right), D(\omega)\right\rangle \mathrm{d} v o l \\
= & \int_{Q_{\lambda}}\left|D Z_{\varepsilon}\right|^{2}+Z_{\varepsilon}^{2}\left\langle D \omega, D\left(\frac{1}{\omega}\right)\right\rangle \mathrm{d} v o l .
\end{aligned}
$$

Hence, using (43), and our assumption that $\frac{|D \omega|^{2}}{\omega^{2}} \leqslant G-\mu$, we deduce

$$
\begin{aligned}
\int_{Q_{\lambda}}\left|D Z_{\varepsilon}\right|^{2} \mathrm{~d} v o l & \leqslant \int_{Q_{\lambda}} \frac{Z_{\varepsilon}^{2}}{\omega^{2}}|D \omega|^{2} \mathrm{~d} v o l+\mu \int_{Q_{\lambda}}\left[\left(u_{\lambda}-u\right)^{+}\right]^{2} \omega^{2} \mathrm{~d} v o l \\
& \leqslant(G-\mu) \int_{Q_{\lambda}} Z_{\varepsilon}^{2} \mathrm{~d} v o l+\mu \int_{Q_{\lambda}}\left[\left(u_{\lambda}-u\right)^{+}\right]^{2} \omega^{2} \mathrm{~d} v o l \\
& \leqslant(G-\mu) \int_{Q_{\lambda}} Z_{\varepsilon}^{2} \mathrm{~d} v o l+\mu \int_{Q_{\lambda}}\left[\left(u_{\lambda}-u\right)^{+}\right]^{2} \omega^{2} \mathrm{~d} v o l \\
& \leqslant(G-\mu) \int_{Q_{\lambda}}\left[\left(u_{\lambda}-u\right)^{+}\right]^{2} \omega^{2} \mathrm{~d} v o l+\mu \int_{Q_{\lambda}}\left[\left(u_{\lambda}-u\right)^{+}\right]^{2} \omega^{2} \mathrm{~d} v o l \\
& =G \int_{Q_{\lambda}}\left[\left(u_{\lambda}-u\right)^{+}\right]^{2} \omega^{2} \mathrm{~d} v o l .
\end{aligned}
$$


Letting $\varepsilon \rightarrow 0$ we see that

$$
\int_{Q_{\lambda}}\left|D\left[\left(u_{\lambda}-u\right)^{+} \omega\right]\right|^{2} \mathrm{~d} v o l \leqslant G \int_{Q_{\lambda}}\left[\left(u_{\lambda}-u\right)^{+}\right]^{2} \omega^{2} \mathrm{~d} v o l .
$$

Using the Sobolev inequality as before, we conclude that $\left(u_{\lambda}-u\right)^{+} \equiv 0$, i.e. $u_{\lambda} \leqslant u$ in $Q_{\lambda}$.

Steps 2 and 3 are proved just as in case (a).

\subsection{Proof of Theorem 12}

Once more, we set $\Lambda=\left\{t \in \mathbb{R}: \forall \tau>t, u \geqslant u_{\tau}\right.$ in $\left.Q_{\tau}\right\}$, the proof consists of the same 3 steps and we only need to give the proof of step 1 since the other two are analogous to those given in the proof of Theorem 11 .

Step 1: $\Lambda$ is nonempty.

As above, we define $\lambda_{0}$ (see (41)) and take $\lambda>\lambda_{0}$. For any $\varepsilon>0$ we take $\left(u_{\lambda}-u-\varepsilon\right)^{+}$ as test function. A direct computation, using condition (36), yields

$$
\begin{aligned}
\int_{Q_{\lambda}}\left|D\left(u_{\lambda}-u-\varepsilon\right)^{+}\right|^{2} \mathrm{~d} v o l & =\int_{Q_{\lambda}}\left(f\left(u_{\lambda}\right)-f(u)\right)\left(u_{\lambda}-u-\varepsilon\right)^{+} \mathrm{d} v o l \\
& \leqslant \int_{Q_{\lambda}}\left(G+C\left(u_{\lambda}\right)^{\alpha}\right)\left(u_{\lambda}-u\right)\left(u_{\lambda}-u-\varepsilon\right)^{+} \mathrm{d} v o l \\
& \leqslant \int_{Q_{\lambda}}\left(G+C\left(u_{\lambda}\right)^{\alpha}\right)\left[\left(u_{\lambda}-u\right)^{+}\right]^{2} \mathrm{~d} v o l .
\end{aligned}
$$

We remark that there exists a constant $C^{\prime}$ s.t.

$$
\left(G+C\left(u_{\lambda}\right)^{\alpha}\right)\left[\left(u_{\lambda}-u\right)^{+}\right]^{2} \leqslant C^{\prime}\left[\left(u_{\lambda}-u\right)^{+}\right]^{2} \in L^{1}(\mathcal{M}) .
$$

Passing to the limit $\varepsilon \rightarrow 0$ we obtain

$$
\int_{Q_{\lambda}}\left|D\left(u_{\lambda}-u\right)^{+}\right|^{2} \mathrm{~d} v o l \leqslant \int_{Q_{\lambda}}\left(G+C\left(u_{\lambda}\right)^{\alpha}\right)\left[\left(u_{\lambda}-u\right)^{+}\right]^{2} \mathrm{~d} v o l,
$$

and thus

$$
\int_{Q_{\lambda}}\left|D\left(u_{\lambda}-u\right)^{+}\right|^{2} \mathrm{~d} v o l-G \int_{Q_{\lambda}}\left[\left(u_{\lambda}-u\right)^{+}\right]^{2} \mathrm{~d} v o l \leqslant C \int_{Q_{\lambda}}\left(u_{\lambda}\right)^{\alpha}\left[\left(u_{\lambda}-u\right)^{+}\right]^{2} \mathrm{~d} v o l .
$$

Using the Hölder and Sobolev inequalities, we deduce

$$
\begin{aligned}
& \int_{Q_{\lambda}}\left|D\left(u_{\lambda}-u\right)^{+}\right|^{2} \mathrm{~d} v o l-G \int_{Q_{\lambda}}\left[\left(u_{\lambda}-u\right)^{+}\right]^{2} \mathrm{~d} v o l \\
& \quad \leqslant C\left(\int_{Q_{\lambda}}\left(u_{\lambda}\right)^{\frac{\alpha n}{2}} \mathrm{~d} v o l\right)^{\frac{2}{n}}\left(\int_{Q_{\lambda}}\left[\left(u_{\lambda}-u\right)^{+}\right]^{\frac{2 n}{n-2}} \mathrm{~d} v o l\right)^{\frac{n-2}{n}}
\end{aligned}
$$




$$
\begin{aligned}
& \leqslant C^{\prime \prime}\left(\int_{Q_{\lambda}}\left(u_{\lambda}\right)^{\frac{\alpha n}{2}} \mathrm{~d} v o l\right)^{\frac{2}{n}}\left(\int_{Q_{\lambda}}\left|D\left(u_{\lambda}-u\right)^{+}\right|^{2}-G\left[\left(u_{\lambda}-u\right)^{+}\right]^{2} \mathrm{~d} v o l\right) \\
& =C^{\prime \prime}\left(\int_{Q^{\lambda}} u^{\frac{\alpha n}{2}} \mathrm{~d} v o l\right)^{\frac{2}{n}}\left(\int_{Q_{\lambda}}\left|D\left(u_{\lambda}-u\right)^{+}\right|^{2}-G\left[\left(u_{\lambda}-u\right)^{+}\right]^{2} \mathrm{~d} v o l\right) .
\end{aligned}
$$

Since $u \in L^{\alpha n / 2}(\mathcal{M})$,

$$
\int_{Q^{\lambda}} u^{\alpha n / 2} \mathrm{~d} v o l \rightarrow 0, \quad \text { when } \lambda \rightarrow+\infty,
$$

and thus we can choose $\lambda_{2}$ such that

$$
C^{\prime \prime}\left(\int_{Q^{\lambda}} u^{\alpha n / 2} \mathrm{~d} v o l\right)^{2 / n}<1, \quad \forall \lambda>\lambda_{2} .
$$

Thus, assuming $\lambda>\lambda_{2}$, we would have

$$
\int_{Q_{\lambda}}\left|D\left(u_{\lambda}-u\right)^{+}\right|^{2}-G\left[\left(u_{\lambda}-u\right)^{+}\right]^{2} \mathrm{~d} v o l<\int_{Q_{\lambda}}\left|D\left(u_{\lambda}-u\right)^{+}\right|^{2}-G\left[\left(u_{\lambda}-u\right)^{+}\right]^{2} \mathrm{~d} v o l
$$

and therefore,

$$
\int_{Q_{\lambda}}\left|D\left(u_{\lambda}-u\right)^{+}\right|^{2} \mathrm{~d} v o l-G \int_{Q_{\lambda}}\left[\left(u_{\lambda}-u\right)^{+}\right]^{2} \mathrm{~d} v o l=0,
$$

since

$$
\int_{Q_{\lambda}}|D v|^{2} \mathrm{~d} v o l \geqslant G \int_{Q_{\lambda}} v^{2} \mathrm{~d} v o l, \quad \forall v \in H_{0}^{1}\left(Q_{\lambda}\right) .
$$

Using the Sobolev inequality (24), it follows from (44) that

$$
\begin{aligned}
& \frac{1}{K^{2}}\left(\int_{Q_{\lambda}}\left[\left(u_{\lambda}-u\right)^{+}\right]^{\frac{2 n}{n-2}} \mathrm{~d} v o l\right)^{\frac{n-2}{n}} \\
& \quad \leqslant \int_{Q_{\lambda}}\left|D\left(u_{\lambda}-u\right)^{+}\right|^{2} \mathrm{~d} v o l-G \int_{Q_{\lambda}}\left[\left(u_{\lambda}-u\right)^{+}\right]^{2} \mathrm{~d} v o l=0,
\end{aligned}
$$

which yields that $\left(u_{\lambda}-u\right)^{+} \equiv 0$ in $Q_{\lambda}$, i.e. that, as desired, $u_{\lambda} \leqslant u$ in $Q_{\lambda}$.

Steps 2 and 3 are proved just as in Theorem 11.

\subsection{Proof of Theorem 13}

Define $\Lambda=\left\{t \in \mathbb{R}: \forall \tau>t, u \geqslant u_{\tau}\right.$ in $\left.Q_{\tau}\right\}$, as above. As before, the proof consists of the same 3 steps and we only need to give the proof of step 1 . 
Step 1: $\Lambda$ is nonempty.

We start by remarking that, since we assume $u \in H^{1}(\mathcal{M})$, we can take $\left(u_{\lambda}-u\right)^{+}$ directly as test function (as in Theorem 5). Hence, using condition (37), we obtain

$$
\begin{aligned}
\int_{Q_{\lambda}}\left|D\left(u_{\lambda}-u\right)^{+}\right|^{2} \mathrm{~d} v o l & =\int_{Q_{\lambda}}\left(-\Delta_{\mathcal{M}}\left(u_{\lambda}-u\right)\right)\left(u_{\lambda}-u\right)^{+} \\
& =\int_{Q_{\lambda}}\left(f\left(u_{\lambda}\right)-f(u)\right)\left(u_{\lambda}-u\right)^{+} \mathrm{d} v o l \\
& \leqslant \int_{Q_{\lambda}}\left(G+C\left(u_{\lambda}\right)^{\alpha}\right)\left[\left(u_{\lambda}-u\right)^{+}\right]^{2} \mathrm{~d} v o l \\
& \leqslant G \int_{Q_{\lambda}}\left[\left(u_{\lambda}-u\right)^{+}\right]^{2} \mathrm{~d} v o l+C \int_{Q_{\lambda}}\left(u_{\lambda}\right)^{\alpha}\left[\left(u_{\lambda}-u\right)^{+}\right]^{2} \mathrm{~d} v o l .
\end{aligned}
$$

Using the Hölder and Sobolev inequalities, it follows that

$$
\begin{aligned}
& \int_{Q_{\lambda}}\left|D\left(u_{\lambda}-u\right)^{+}\right|^{2} \mathrm{~d} v o l \\
& \leqslant G \int_{Q_{\lambda}}\left[\left(u_{\lambda}-u\right)^{+}\right]^{2} \mathrm{~d} v o l+C\left(\int_{Q_{\lambda}}\left(u_{\lambda}\right)^{\frac{\alpha n}{2}} \mathrm{~d} v o l\right)^{\frac{2}{n}}\left(\int_{Q_{\lambda}}\left[\left(u_{\lambda}-u\right)^{+}\right]^{\frac{2 n}{n-2}} \mathrm{~d} v o l\right)^{\frac{n-2}{n}} \\
& \leqslant G \int_{Q_{\lambda}}\left[\left(u_{\lambda}-u\right)^{+}\right]^{2} \mathrm{~d} v o l+C_{1}\left(\int_{Q_{\lambda}}\left(u_{\lambda}\right)^{\frac{\alpha n}{2}} \mathrm{~d} v o l\right)^{\frac{2}{n}} \\
& \quad \times\left(\int_{Q_{\lambda}}\left|D\left(u_{\lambda}-u\right)^{+}\right|^{2}-G\left[\left(u_{\lambda}-u\right)^{+}\right]^{2} \mathrm{~d} v o l\right) .
\end{aligned}
$$

Thus,

$$
\left(1-C\left(\int_{Q_{\lambda}}\left(u_{\lambda}\right)^{\frac{\alpha n}{2}} \mathrm{~d} v o l\right)^{\frac{2}{n}}\right)\left(\int_{Q_{\lambda}}\left|D\left(u_{\lambda}-u\right)^{+}\right|^{2}-G\left[\left(u_{\lambda}-u\right)^{+}\right]^{2} \mathrm{~d} v o l\right) \leqslant 0 .
$$

As above, we can choose $\lambda_{2} \in \mathbb{R}$ s.t.

$$
C\left(\int_{Q_{\lambda}}\left(u_{\lambda}\right)^{\frac{\alpha n}{2}} \mathrm{~d} v o l\right)^{\frac{2}{n}}<1 \forall \lambda>\lambda_{2} .
$$

Then, for any $\lambda>\lambda_{2}$

$$
\int_{Q_{\lambda}}\left|D\left(u_{\lambda}-u\right)^{+}\right|^{2} \mathrm{~d} v o l-G \int_{Q_{\lambda}}\left[\left(u_{\lambda}-u\right)^{+}\right]^{2} \mathrm{~d} v o l \leqslant 0 .
$$

Together with the Sobolev inequality, this implies that 


$$
\begin{aligned}
& \frac{1}{K^{2}}\left(\int_{Q_{\lambda}}\left[\left(u_{\lambda}-u\right)^{+}\right]^{\frac{2 n}{n-2}} \mathrm{~d} v o l\right)^{\frac{n-2}{n}} \\
& \quad \leqslant \int_{Q_{\lambda}}\left|D\left(u_{\lambda}-u\right)^{+}\right|^{2} \mathrm{~d} v o l-G \int_{Q_{\lambda}}\left[\left(u_{\lambda}-u\right)^{+}\right]^{2} \mathrm{~d} v o l \leqslant 0 .
\end{aligned}
$$

Therefore, $\left(u_{\lambda}-u\right)^{+} \equiv 0$ in $Q_{\lambda}$ and thus $\left(\lambda_{2},+\infty\right) \subset \Lambda$.

As for steps 2 and 3, they are proved just as in Theorem 11 .

\subsection{Passing from directional to radial symmetry}

If our manifold has an appropriate structure it is possible to pass from directional symmetry results like those obtained in Theorems 10-13, to the corresponding full radial symmetry results like Theorems $6-9$. We will show here the procedure to obtain the full radial symmetry results in $\mathbb{H}^{n}$ using the directional symmetry results given by Theorems 10-13 (it is analogous to the standard method used in $\mathbb{R}^{n}$ ).

We start by considering $n$ orthogonal directions in $\mathbb{H}^{n}$ and obtain the symmetry along these directions using the appropriate result (Theorem 10 to prove Theorem 6 , Theorem 11 to prove Theorem $7, \ldots$ ). The $n$ symmetry hypersurfaces obtained in this way (corresponding to the $n$ directions considered), which we denote by $W_{1}, \ldots, W_{n}$, are orthogonal to each other and their intersection is a unique point $x_{0} \in \mathcal{M}$.

Let $P_{i}$ be the orthogonal projection onto $W_{i}$ (projection along the transformation group associated with the $i$ th direction considered). Then $\forall x \in \mathcal{M}$ and $\forall i=1, \ldots, n$,

$$
u(x) \leqslant u\left(P_{i}(x)\right), \text { with equality iff } x=P_{i}(x),
$$

since our directional symmetry results imply that the function $u$ is strictly increasing along group action trajectories, up to the central position $\left(W_{i}\right)$, unless $u$ is constant, in which case the radial symmetry is trivial. Henceforth we exclude this trivial case. Indeed, in step 1 of the proofs of our directional symmetry results above, the strong maximum principle implies that $\forall \lambda \in \Lambda, X(u)(x)<0, \forall x \in U_{\lambda}$.

Consequently, since $x_{0}=P_{1} P_{2} \ldots P_{n}(x)$,

$$
u(x) \leqslant u\left(P_{1} P_{2} \ldots P_{n}(x)\right)=u\left(x_{0}\right) .
$$

Thus, $x_{0}$ is the unique maximum point for $u$.

Now, consider any other arbitrarily chosen direction in $\mathbb{H}^{n}$. Our theorems yield the directional symmetry along this direction (relative to some hypersurface $W$ ). We claim that $W$ passes through $x_{0}$. In fact, let $P$ denote the projection, along this direction, onto $W$. Then, by (45) for $P$, if $x_{0} \notin W$, it follows that $u\left(P\left(x_{0}\right)\right)>u\left(x_{0}\right)$, contradicting (46).

Working with stereographic coordinates in $\mathbb{H}^{n}$, just to fix ideas, and taking advantage of the fact that $\mathbb{H}^{n}$ is a homogeneous manifold, w.l.o.g. we may suppose $x_{0}$ is the origin. Then, what our argument above tells us is that $u$ is symmetric with respect to every hyperplane passing through the origin, and thus, $u$ is radially symmetric in the usual sense.

The fact that the solutions are also strictly radially decreasing is obtained just as in the $\mathbb{R}^{n}$ case. 
Remark. - For product spaces like $\mathbb{H}^{n} \times S^{m}$, with $n>m$, or $\mathbb{H}^{n} \times \mathbb{R}^{k}$, we have $B<0$ in (24) and thus our theorems apply and yield that a positive solution $u$ is necessarily radially symmetric along the $\mathbb{H}^{n}$ factor (like in Example 4.5 of [2]). The same is true for product spaces $\mathbb{H}^{n} \times \mathcal{M}$, where $\mathcal{M}$ is a compact manifold, as long as $n$ is sufficiently large (so that $B$ becomes negative) and the product manifold is simply connected and uniformly flat.

When $B=0$, which is the case of $\mathbb{H}^{n} \times S^{n}$, we should have the same type of results as in $\mathbb{R}^{n}$.

\section{More general manifolds}

In this section we will work in a more general setting than in Section 4. The assumptions on the Riemann curvature tensor will be the same, in order to have the Sobolev inequality (24), but we no longer assume the nice group structure associated with the foliation that we had in the previous section. The setting is somewhat similar to the one considered in the general case of [2] and, in this spirit, although here we suppose $\mathcal{M}$ to be a noncompact manifold without boundary, our results should also be valid in the case $\partial \mathcal{M} \neq \varnothing$ as long as we ask $u=0$ on $\partial \mathcal{M}$.

Our purpose here is to show that, although it is technically more complicated, it is still possible to obtain the same type of result as the ones obtained above. As an illustration, we will state and prove the equivalent of Theorem 10 in this setting.

\subsection{Foliation conditions}

The foliation conditions we will assume here are the following: there exists a family of isometries $I_{t}: \mathcal{M} \rightarrow \mathcal{M}, t \in \mathbb{R}$, which is $C^{1}$ in $t$, and such that there is a family of pairwise disjoint hypersurfaces $U_{t} \subset \mathcal{M}$ such that $I_{t}(x)=x \Leftrightarrow x \in U_{t}$, i.e. $U_{t}$ is the invariant hypersurface under the action of $I_{t}$. The manifold $\mathcal{M}$ is foliated by these surfaces, i.e. $\mathcal{M}=\bigcup_{t \in \mathbb{R}} U_{t}$.

Let $Q_{t_{1}}=\bigcup_{-\infty<t<t_{1}} U_{t}$ and $Q^{t_{1}}=\bigcup_{t_{1}<t<+\infty} U_{t}$. Then, $I_{t}\left(Q_{t}\right) \subset Q^{t}$ and $I_{t}\left(Q^{t}\right) \subset Q_{t}$, for all $t \in \mathbb{R}$. Furthermore, we suppose that the $Q_{t}$ are connected for all $t \in \mathbb{R}$ (so that we can apply the strong maximum principle as in the previous settings). For $t \in \mathbb{R}$ and $x \in Q_{t}$ we define $x_{t}=I_{t}(x)$ and $u_{t}(x)=u\left(x_{t}\right)$. The function $t(x)$ assigns to each $x \in \mathcal{M}$, the unique $t \in \mathbb{R}$ s.t. $x \in U_{t}$. It is assumed to be a continuous function.

\subsection{Main theorem}

In the following theorem, which is a generalization of Theorem 10 to this setting, we suppose that $u$ is a $C^{1}$ weak solution of (30) and (31), where $f$ is assumed to be locally Lipschitz in $(0,+\infty)$.

THEOREM 14. - Under the above assumptions, if there exists $\delta>0$ s.t. $f$ is decreasing in $(0, \delta)$, then $u$ is symmetric relative to some hypersurface $U_{\bar{\lambda}}$, i.e. $\exists \bar{\lambda} \in \mathbb{R}$ s.t. $u_{\bar{\lambda}}(x) \equiv u(x), \forall x \in Q_{\bar{\lambda}}$. 
Proof. - The proof will be done using a generalized moving planes method (in the spirit of [2]). We will structure it into the same three steps as in the proofs of the previous theorems. We start by defining $\Lambda=\left\{t \in \mathbb{R}: \forall \tau>t, u \geqslant u_{\tau}\right.$ in $\left.Q_{\tau}\right\}$.

Step 1: $\Lambda$ is nonempty.

Since, by (31), $u \rightarrow 0$ at infinity, given a fixed point $x_{1} \in \mathcal{M}$, there exists $R_{1}>0$ s.t.

$$
u<\delta \quad \text { in } \mathcal{M} \backslash \bar{B}\left(x_{1}, R_{1}\right),
$$

where $\bar{B}\left(x_{1}, R_{1}\right)$ denoted the closed geodesic ball of center $x_{1}$ and radius $R_{1}$. Let $t: \mathcal{M} \rightarrow \mathbb{R}$ be our foliation parameter, which is a continuous function. Then, $t\left(\bar{B}\left(x_{1}, R_{1}\right)\right)$ is a compact subset of $\mathbb{R}$ and thus, $\exists \sigma \in \mathbb{R}$ s.t. $t\left(\bar{B}\left(x_{1}, R_{1}\right)\right) \cap[\sigma, \infty)=\emptyset$.

We claim that $\sigma \in \Lambda$. In fact, given any $\lambda \in[\sigma, \infty)$, and for every $\varepsilon>0$, we can take

$$
w_{\varepsilon}:=\left(u_{\lambda}-u-\varepsilon\right)^{+}
$$

as test function since it belongs to $H_{0}^{1}\left(Q_{\lambda}\right)$ (because $u$ is $C^{1}$ and tends to zero at infinity). This yields,

$$
\int_{Q_{\lambda}}-\Delta\left(u-u_{\lambda}\right) w_{\varepsilon} \mathrm{d} v o l=\int_{Q_{\lambda}}\left(f(u)-f\left(u_{\lambda}\right)\right) w_{\varepsilon} \mathrm{d} v o l,
$$

where, as before, we used the fact that $u_{\lambda}$ is also a solution of our problem since the equation is invariant by the isometry $I_{\lambda}$. On the one hand, for the 1.h.s. we have that

$$
\int_{Q_{\lambda}}-\Delta\left(u-u_{\lambda}\right) w_{\varepsilon} \mathrm{d} v o l=\int_{Q_{\lambda}}\left\langle D\left(u-u_{\lambda}\right), D w_{\varepsilon}\right\rangle \mathrm{d} v o l=\int_{Q_{\lambda}}\left|D w_{\varepsilon}\right|^{2} \mathrm{~d} v o l .
$$

On the other hand, for the r.h.s.

$$
\int_{Q_{\lambda}}\left(f(u)-f\left(u_{\lambda}\right)\right) w_{\varepsilon} \mathrm{d} v o l \leqslant 0,
$$

since, when $w_{\varepsilon}(x) \neq 0$ in $Q_{\lambda}$, we have $\delta>u_{\lambda}(x)>u(x)+\varepsilon>0$, and $f$ is decreasing in $(0, \delta)$. Therefore, it follows from (47) that

$$
\int_{Q_{\lambda}}\left|D w_{\varepsilon}\right|^{2} \mathrm{~d} v o l \leqslant 0,
$$

and hence, $w_{\varepsilon}=0$ (since it is a constant and at infinity it is zero), i.e. $u_{\lambda} \leqslant u+\varepsilon$ in $Q_{\lambda}$. Passing to the limit when $\varepsilon \rightarrow 0$, it follows that $u_{\lambda} \leqslant u$ in $Q_{\lambda}$, and this for all $\lambda \geqslant \sigma$. Thus, $\sigma \in \Lambda$, as claimed.

Step 2: $\Lambda$ is bounded from below.

We proceed as in step 2 of the proof of Theorem 11: using the fact that $\lim _{\lambda \rightarrow-\infty} \sup _{Q_{\lambda}} u=0$, we can choose some $\sigma_{1}$ s.t. $\sup _{Q_{\sigma_{1}}} u<\left(\sup _{\mathcal{M}} u\right) / 2$. Hence, all $\lambda \in\left(-\infty, \sigma_{1}\right)$ do not belong to $\Lambda$. Therefore, $\Lambda$ is bounded from below, and we let

$$
\bar{\lambda}:=\inf \Lambda \text {. }
$$


Step 3: $u \equiv u_{\bar{\lambda}}$ in $Q_{\bar{\lambda}}$.

We will proceed by contradiction. By continuity, $u \geqslant u_{\bar{\lambda}}$ in $Q_{\bar{\lambda}}$. If $u \neq u_{\bar{\lambda}}$, then

$$
\forall y \in Q_{\bar{\lambda}}: u(y)>u_{\bar{\lambda}}(y) .
$$

Indeed, we have that for any $\lambda,-\Delta\left(u-u_{\lambda}\right)=a\left(u-u_{\lambda}\right)$ in $Q_{\lambda}$, where

$$
a=a_{\lambda}(x):= \begin{cases}0, & \text { if } u(x)=u_{\lambda}(x), \\ \frac{f(u(x))-f\left(u_{\lambda}(x)\right)}{u(x)-u_{\lambda}(x)}, & \text { if } u(x) \neq u_{\lambda}(x) .\end{cases}
$$

Since $a_{\bar{\lambda}}(x)$ is a locally bounded function ( $f$ is locally Lipschitz and $u$ is positive and bounded), and $Q_{\bar{\lambda}}$ is connected, it follows from (48) and the standard strong maximum principle (see, for instance, [2] Theorem 3 ) that

$$
u>u_{\bar{\lambda}} \quad \text { in } Q_{\bar{\lambda}} .
$$

Let $x_{0} \in U_{\bar{\lambda}}$ and choose $R>0$ s.t.

$$
\bar{B}\left(x_{1}, R_{1}\right) \subset B\left(x_{0}, R\right) .
$$

For $\lambda \in \mathbb{R}$, let $V_{\lambda}:=U_{\lambda} \cap \bar{B}\left(x_{0}, 3 R\right)$ and $\tilde{V}_{\bar{\lambda}}:=U_{\bar{\lambda}} \cap \bar{B}\left(x_{0}, 2 R\right) \tilde{V}_{\bar{\lambda}}$ is compact since $U_{\bar{\lambda}}$ is closed and $\bar{B}\left(x_{0}, 2 R\right)$ is compact. Therefore, we can cover $\tilde{V}_{\bar{\lambda}}$ by a finite number of balls $B_{i}$ with center in $\tilde{V}_{\bar{\lambda}}, i=1, \ldots, N$, such that

$$
\operatorname{radius}\left(B_{i}\right)<\min \left\{\rho, \frac{R}{2}\right\},
$$

where $\rho>0$ is the injectivity radius of $\mathcal{M}$. Since $\tilde{V}_{\bar{\lambda}}$ is compact and is contained in $B:=\bigcup_{i=1}^{N} B_{i}$, which is an open set, $\operatorname{dist}\left(\tilde{V}_{\bar{\lambda}}, \partial B\right)>0$. Thus, by the continuity of our foliation,

$$
\exists \lambda_{1}<\bar{\lambda}: V_{\lambda} \cap B\left(x_{0}, 2 R\right) \subset B, \quad \forall \lambda_{1}<\lambda<\bar{\lambda} .
$$

On each of the balls $B_{i}$, we have a Poincaré inequality: $\forall v \in H^{1}\left(B_{i}\right)$ s.t. $f_{B_{i}} v=0$,

$$
\int_{B_{i}} v^{2} \leqslant\left|B_{i} \cap \operatorname{supp}(v)\right|^{2 / n}\left(\int_{B_{i}} v^{\frac{2 n}{n-2}} \mathrm{~d} v o l\right)^{\frac{n-2}{n}} \leqslant C_{i}\left|B_{i} \cap \operatorname{supp}(v)\right|^{2 / n} \int_{B_{i}}|D v|^{2} .
$$

Here $C_{i}$ is a constant (see, for instance [12] Theorem 5.11.2). Let $C:=\max C_{i}, \ell$ be the Lipschitz constant for $f$ in the interval $\left(\min _{\bar{B}\left(x_{0}, 2 R\right)} u, \max _{\bar{B}\left(x_{0}, 2 R\right)} u\right)$, and $H_{\tau}:=$ $\bigcup_{\tau<\lambda<\bar{\lambda}} V_{\lambda}$, be the band in $\bar{B}\left(x_{0}, 3 R\right)$ between $V_{\tau}$ and $V_{\bar{\lambda}}$. We may choose $\lambda_{2} \in\left(\lambda_{1}, \bar{\lambda}\right)$ s.t.

$$
8 C \ell N\left|H_{\lambda_{2}}\right|^{2 / n}<1 .
$$

Let $K:=\bar{Q}_{\lambda_{2}} \cap \bar{B}\left(x_{0}, 3 R\right)$, which is a compact set. By the continuity of $u$ and of the foliation (which includes the family of reflections), since $u>u_{\bar{\lambda}}$ on $K$,

$$
\exists \lambda_{3} \in\left(\lambda_{2}, \bar{\lambda}\right) \text { s.t. } \forall \lambda \in\left[\lambda_{3}, \bar{\lambda}\right] \quad u>u_{\lambda} \text { on } K .
$$


Fix any $\lambda \in\left(\lambda_{3}, \bar{\lambda}\right)$ and, for every $\varepsilon>0$, take as test function

$$
w_{\varepsilon}:=\left(u_{\lambda}-u-\varepsilon\right)^{+} .
$$

We notice that $\forall \varepsilon>0, w_{\varepsilon} \equiv 0$ on $K$. Moreover, from (50) and (51) it follows that $\forall \lambda \in\left(\lambda_{1}, \bar{\lambda}\right]$ and $\forall x \in Q_{\lambda} \backslash B\left(x_{0}, 2 R\right)$ we have dist $\left(x_{\lambda}, x_{0}\right)>R$ and thus, $u(x)<\delta$ and $u_{\lambda}(x)<\delta$. Therefore,

$$
\begin{aligned}
\int_{Q_{\lambda}}-\Delta\left(u-u_{\lambda}\right) w_{\varepsilon} \mathrm{d} v o l & =\int_{Q_{\lambda}}\left\langle D\left(u-u_{\lambda}\right), D w_{\varepsilon}\right\rangle \mathrm{d} v o l=\int_{Q_{\lambda}}\left|D w_{\varepsilon}\right|^{2} \mathrm{~d} v o l \\
& =\int_{Q_{\lambda} \backslash K}\left|D w_{\varepsilon}\right|^{2} \mathrm{~d} v o l .
\end{aligned}
$$

On the other hand, from our equation it follows that, since $Q_{\lambda} \backslash K \subset\left(\left(H_{\lambda_{2}} \backslash H_{\lambda}\right) \cap\right.$ $\left.B\left(x_{0}, 2 R\right)\right) \cup\left(Q_{\lambda} \cap\left(\bar{B}\left(x_{0}, R\right)\right)^{c}\right)$,

$$
\begin{aligned}
& \int_{Q_{\lambda}}-\Delta\left(u-u_{\lambda}\right) w_{\varepsilon} \mathrm{d} v o l=\int_{Q_{\lambda}}\left(f(u)-f\left(u_{\lambda}\right)\right) w_{\varepsilon} \mathrm{d} v o l \\
& \leqslant \int_{\left(H_{\lambda_{2}} \backslash H_{\lambda}\right) \cap B\left(x_{0}, 2 R\right)}\left|f(u)-f\left(u_{\lambda}\right)\right| w_{\varepsilon} \mathrm{d} v o l \\
& +\int_{Q_{\lambda} \cap\left(\bar{B}\left(x_{0}, R\right)\right)^{c}}\left(f(u)-f\left(u_{\lambda}\right)\right) w_{\varepsilon} \mathrm{d} v o l \\
& \leqslant \int_{\left(H_{\lambda_{2}} \backslash H_{\lambda}\right) \cap B\left(x_{0}, 2 R\right)}\left|f(u)-f\left(u_{\lambda}\right)\right| w_{\varepsilon} \mathrm{d} v o l,
\end{aligned}
$$

where, in the last inequality, we used the fact that on $\operatorname{supp}\left(w_{\varepsilon}\right) \cap\left(\bar{B}\left(x_{0}, R\right)\right)^{c}$ we have $\delta>u_{\lambda}>u>0$ and $f(u)-f\left(u_{\lambda}\right) \leqslant 0$. Moreover, $\left|f(u)-f\left(u_{\lambda}\right)\right| \leqslant \ell\left|u_{\lambda}-u\right|$, and $w_{\varepsilon} \leqslant\left(u_{\lambda}-u\right)^{+}$on $B\left(x_{0}, 2 R\right)$. Therefore, (56) and (57) yield

$$
\int_{Q_{\lambda}}\left|D w_{\varepsilon}\right|^{2} \mathrm{~d} v o l \leqslant \ell \int_{\left(H_{\lambda_{2}} \backslash H_{\lambda}\right) \cap B\left(x_{0}, 2 R\right)}\left[\left(u_{\lambda}-u\right)^{+}\right]^{2} \mathrm{~d} v o l .
$$

Letting $\varepsilon \rightarrow 0$, we obtain

$$
\begin{aligned}
\int_{Q_{\lambda}}\left|D\left(u_{\lambda}-u\right)^{+}\right|^{2} \mathrm{~d} v o l & \leqslant \ell \int_{\left(H_{\lambda_{2}} \backslash H_{\lambda}\right) \cap B\left(x_{0}, 2 R\right)}\left[\left(u_{\lambda}-u\right)^{+}\right]^{2} \mathrm{~d} v o l \\
& \leqslant \ell \sum_{i=1}^{N} \int_{\left(H_{\lambda_{2}} \backslash H_{\lambda}\right) \cap B_{i}}\left[\left(u_{\lambda}-u\right)^{+}\right]^{2} \mathrm{~d} v o l .
\end{aligned}
$$


To apply Poincaré inequality (53) we will consider the following extensions of $\left(u_{\lambda}-u\right)^{+}$to $B_{i}$

$$
v_{i}:= \begin{cases}\left(u_{\lambda}-u\right)^{+}, & \text {in } B_{i} \cap Q_{\lambda}, \\ 0, & \text { in }\left(B_{i} \cap H_{\lambda}\right) \cup I_{\bar{\lambda}}\left(B_{i} \cap H_{\lambda}\right), \\ -\left(u_{\lambda}-u\right)^{+}\left(I_{\bar{\lambda}}(x)\right), & \text { if } x \in I_{\bar{\lambda}}\left(B_{i} \cap Q_{\lambda}\right) .\end{cases}
$$

Using these $v_{i},(58)$ and (53) we obtain

$$
\begin{aligned}
\int_{Q_{\lambda}}\left|D\left(u_{\lambda}-u\right)^{+}\right|^{2} \mathrm{~d} v o l & \leqslant \ell \sum_{i=1}^{N} \int_{B_{i}} v_{i}^{2} \mathrm{~d} v o l \\
& \leqslant \ell \sum_{i=1}^{N} C_{i}\left|B_{i} \cap \operatorname{supp}\left(v_{i}\right)\right|^{2 / n} \int_{B_{i}}\left|D v_{i}\right|^{2} \mathrm{~d} v o l \\
& \leqslant 2 \ell \sum_{i=1}^{N} C_{i}\left|B_{i} \cap \operatorname{supp}\left(v_{i}\right)\right|^{2 / n} \int_{B_{i} \cap Q_{\bar{\lambda}}}\left|D v_{i}\right|^{2} \mathrm{~d} v o l \\
& \leqslant 2 \ell \sum_{i=1}^{N} C_{i} 2^{2 / n}\left|B_{i} \cap Q_{\bar{\lambda}} \cap \operatorname{supp}\left(v_{i}\right)\right|^{2 / n} \int_{B_{i} \cap Q_{\bar{\lambda}}}\left|D v_{i}\right|^{2} \mathrm{~d} v o l .
\end{aligned}
$$

Since $n \geqslant 1$, we have $2^{2 / n} \leqslant 4$. Furthermore, $\operatorname{supp}\left(v_{i}\right) \cap Q_{\bar{\lambda}} \subset H_{\lambda_{2}} \backslash H_{\lambda}$. Thus,

$$
\begin{aligned}
\int_{Q_{\lambda}}\left|D\left(u_{\lambda}-u\right)^{+}\right|^{2} \mathrm{~d} v o l & \leqslant 8 \ell \sum_{i=1}^{N} C_{i}\left|B_{i} \cap H_{\lambda_{2}}\right|^{2 / n} \int_{B_{i} \cap Q_{\lambda}}\left|D v_{i}\right|^{2} \mathrm{~d} v o l \\
& \leqslant 8 \ell C N\left|H_{\lambda_{2}}\right|^{2 / n} \int_{Q_{\lambda}}\left|D\left(u_{\lambda}-u\right)^{+}\right|^{2} \mathrm{~d} v o l .
\end{aligned}
$$

Hence $\left(1-8 \ell C N\left|H_{\lambda_{2}}\right|^{2 / n}\right) \int_{Q_{\lambda}}\left|D\left(u_{\lambda}-u\right)^{+}\right|^{2} \mathrm{~d} v o l \leqslant 0$.

Consequently $u_{\lambda} \leqslant u$ in $Q_{\lambda}$ by (54). Since $\lambda$ was arbitrarily chosen in $\left(\lambda_{2}, \bar{\lambda}\right)$, this would contradict the definition of $\bar{\lambda}$ as $\inf \Lambda$. Therefore, we have $u \equiv u_{\bar{\lambda}}$ in $Q_{\bar{\lambda}}$.

\section{Acknowledgements}

We would like to thank the referee for some useful suggestions about the paper and both the referee and G. Tarantello for (during the submission process) having brought to our attention the existence of references [18] and [19].

\section{REFERENCES}

[1] A. Alexandrov, Uniqueness theorem for surfaces in the large, Vestnik Leningrad Univ. Math. 11 (1956) 5-17.

[2] L. Almeida, Y. Ge, Symmetry results for positive solutions of some elliptic equations on manifolds, Annals Global Anal. Geom. 18 (2000) 153-170. 
[3] H. Berestycki, L.A. Caffarelli, L. Nirenberg, Symmetry for elliptic equations in a half space, in: Boundary Value Problems for Partial Differential Equations and Applications, RMA Res. Notes Appl. Math., Vol. 29, Masson, Paris, 1993, pp. 27-42.

[4] H. Berestycki, L.A. Caffarelli, L. Nirenberg, Inequalities for second order elliptic equations with applications to unbounded domains. I, Duke Math. J. 81 (1996) 467-494.

[5] H. Berestycki, L.A. Caffarelli, L. Nirenberg, Monotonicity for elliptic equations in unbounded Lipshitz domains, Comm. Pure Appl. Math. 50 (1997) 1089-1111.

[6] H. Berestycki, L.A. Caffarelli, L. Nirenberg, Further qualitative properties for elliptic equations in unbounded domains, Ann. Scuola Norm. Sup. Pisa Cl. Sci. 25 (1997) 6994.

[7] H. Berestycki, L. Nirenberg, On the method of moving planes and the sliding method, Bol. Soc. Bras. Mat. 22 (1991) 1-39.

[8] L. Damascelli, F. Pacella, M. Ramaswamy, Symmetry of ground states of $p$-Laplace equations via the moving plane method, Arch. Ration. Mech. Anal. 148 (1999) 291-308.

[9] L. Damascelli, M. Ramaswamy, Symmetry of $C^{1}$ solutions of $p$-Laplace equations in $\mathbb{R}^{N}$, Advanced Nonlinear Studies 1 (2001) 40-64.

[10] B. Gidas, W.M. Ni, L. Nirenberg, Symmetry and related properties via the maximum principle, Comm. Math. Phys. 68 (1979) 209-243.

[11] B. Gidas, W.M. Ni, L. Nirenberg, Symmetry of positive solutions of nonlinear elliptic equations in $\mathbb{R}^{N}$, Adv. Math., Suppl. Stud. 7A (1981) 369-402.

[12] E. Hebey, Introduction à l'analyse non linéaire sur les variétés, Fondations, Diderot Editeur, Paris, 1997.

[13] E. Hebey, Sobolev Spaces on Riemannian Manifolds, Lecture Notes in Mathematics, Vol. 1635, Springer-Verlag, Berlin, 1996.

[14] C. Li, Monotonicity and symmetry of solutions of fully nonlinear elliptic equations on unbounded domains, Comm. Partial Differential Equations 16 (1991) 585-615.

[15] Y. Li, W.M. Ni, Radial symmetry of positive solutions of nonlinear elliptic equations in $\mathbb{R}^{N}$, Comm. Partial Differential Equations 18 (1993) 1043-1054.

[16] J. Serrin, A symmetry problem in potential theory, Arch. Ration. Mech. Anal. 43 (1971) 304-318.

[17] J. Serrin, H. Zou, Symmetry of ground states of quasilinear elliptic equations, Arch. Ration. Mech. Anal. 148 (1999) 265-290.

[18] S. Terracini, Symmetry properties of positive solutions to some elliptic equations with nonlinear boundary conditions, Differential Integral Equations 8 (1995) 1911-1922.

[19] S. Terracini, On positive entire solutions to a class of equations with a singular coefficient and critical exponent, Adv. Differential Equations 1 (1996) 241-264. 